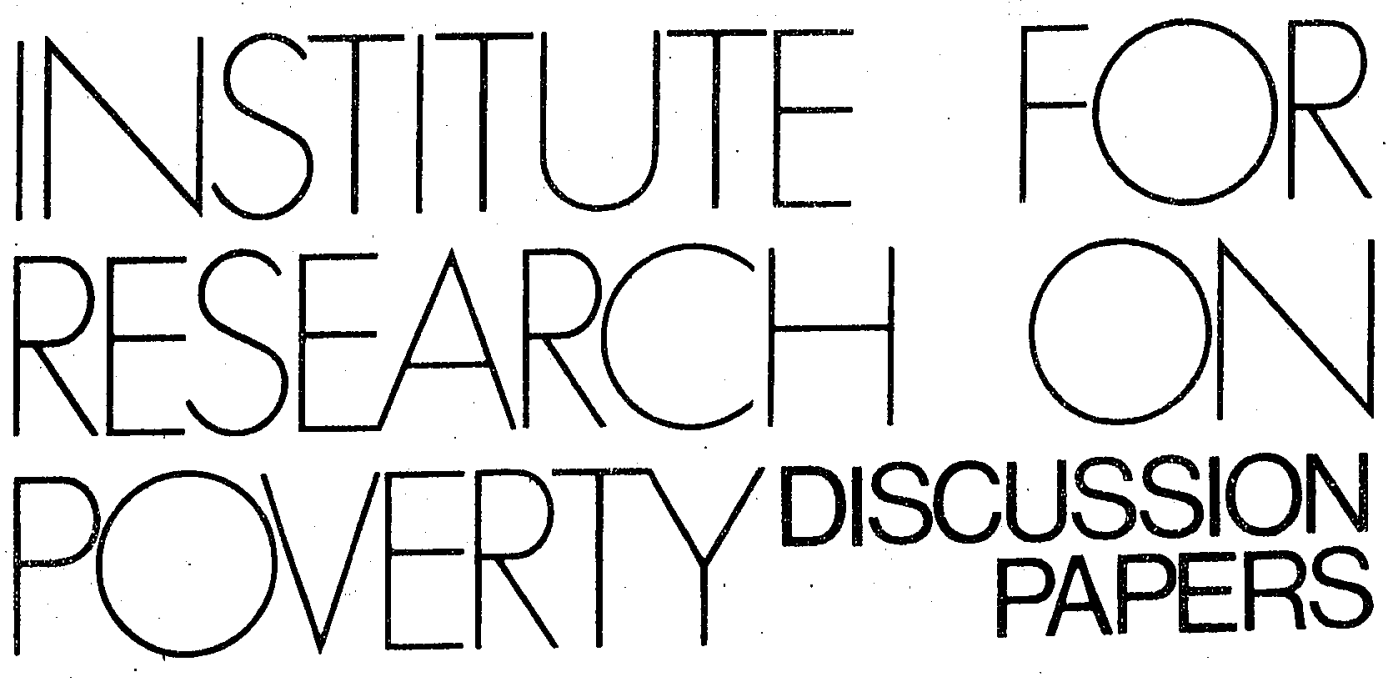

THE REPRESENTATION OF SOCIAL PROCESSES BY MARKOV MODELS

Burton Singer

Seymour Spilerman

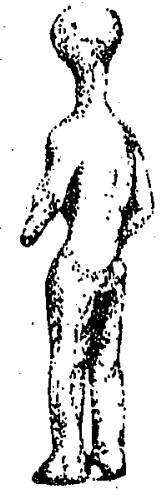




\section{THE REPRESENTATION OF SOCIAL PROCESSES \\ BY MARKOV MODELS *}

Burton Singer

Columbia University
Seymour Spllerman

University of Wisconsin

*The work reported here was supported by grants NSF-GP-31505X and NSF-GS-38574 at Columbia University, and by funds granted to the Institute for Research on Poverty of the UnIversity of Wisconsin by the Office of Economic Opportunity pursuant to the Economic Opportunity Act of 1964. We also acknowledge computation trends from NICHD grant 1-P01-HD05876. Mr. Ear1 Kinnemouth assisted ably with the computations. Any opinions expressed are those of the authors. 


\section{CONTENTS}

1. Introduction

2. Mathematical Preliminaries and Examples

3. Embeddability of $\hat{\mathrm{P}}$

3.1 Necessary Conditions

3.2 The Matrix Equation $\hat{P}=e^{Q}$

3.3 Summary of Steps to Determine Embeddability

4. Multiple Solutions of $\hat{P}=e^{Q}$

4.1 Conceptual Overview

4.2 How Multiple Versions of $\log \hat{P}(t) \varepsilon Q$ Arlse

4.3 Summary of Correspondence between Eigenvalue Characteristics and Number of Matrices $Q \varepsilon \underline{\underline{Q}}$.

5. Testing Strategies

\subsection{Identification of Structural Parameters}

5.2 Sampling Error and Data Noise

6. Conclusions

Appendix 1. Exploring A Continuum 
THE REPRESENTATION OF SOCIAL PROCESSES BY MARKOV MODELS

\section{INTRODUCTION}

Markov models provide a convenient framework for analyzing the structural mechanisms which underly social change, and for extrapolating shifts In the state distribution of a population. For reviews of applications and discussions of some pertinent mathematical issues, the reader is referred to Boudon (1973), Bartholomew (1973), and Singer and Spilerman (1974). A.though most commonly employed in the study of mobllity, Markov models have been applied to diverse substantive topics; they have been used, for instance, to study the influence of group norms on conformity (Cohen 1963), to measure distance in social networks (Beshers and Laumann 1967), and to analyze recidivism among delinquent juveniles (Wolfgang, Figlio, and Sellin 1972). The attractiveness of this mathematical formulation derives from the fact that it permits a researcher to focus upon the dynamic properties of a social process, and ascertain the long-range consequences of particular institutional arrangements. An instructive example of this sort of inquiry is provided by Lieberson and Fuguitt (1967).

Several technical issues which relate to the sensitfve use of Markov modeis have only recently begun to receive an amount of attention that is commensurate with their importance. One matter concerns the phenomenon of population heterogeneity. In the initial attempts at modeling mobility processes by time-stationary Markov chains, socially heterogeneous populations were treated as though a single transition rule governed the movements of all individuals. Special kinds of discrepancles that were observed between the empirical data and predictions from these one-type Markov models were suggestive about the form of 
stochastlc process which might provide a more realistic theoretical framework in which to view mobility (Blumen, Kogan, and McCarthy 1955). The main attempts at modifying the Markov model so it would provide a suitable description of movements by a heterogeneous population have involved viewing the population as consisting of a mixture of independent Markov processes, one for each individual or each distinct soctal group (McFarland 1970; Ginsberg 1971; Spllerman 1972a; 1972b; Singer and Sp1lerman 1974).

A second issue concerns strategies for testing whether empirical observations are, in fact, compatible with an assumed class of models, such as general finite-state Markov processes, or a subset of them, such as birth and death processes. An example of this sort of inquiry is presented in Singer and Spilerman (1974, pp. 360-363), where an observed 2-step matrix ${ }^{1} \hat{P}(2)$--representing occupational change between grandfathers' and respondents' generations--was examined for compatibility with a stationary discrete-time Markov structure. Conceptual1y, the problem is to decide whether the empirically determined matrix could have arisen via the evolution of the postulated process. Stated technically, it is to ascertain whether there exists a one-step transition matrix $P(1)$-which would be identified with grandfather to father transitions or, equivalently, with father to son transitions--such that $\hat{P}(2)=[P(1)]^{2}$. Where the answer is negative, it would be incorrect to predict future population distributions from a Markov model, ${ }^{2}$ such as by raising the observed matrix to integer powers.

The same kind of 1ssue must be faced with respect to compatibility of observed data with other model structures, and it is this fundamental sort of inquiry that we address in the present paper. We will 
concentrate on conditions for compatibility with a finite-state continuoustime Markov process, a mathematical structure which holds special interest for two reasons. First, although discrete-time formulations have been used in most applications of Markov models, the empirical processes under consideration commonly evolve continuously, and the appropriate technical apparatus would be a continuous-time model (Coleman 1964a, p. 129). The reason for the greater popularity of the discrete-time structure stems from its simpler mathematical nature, not from considerations of verisimilitude. Second, continuous-time Markov processes provide the underlying mathematical framework for James S. Coleman's (1964a) influential volume In mathematical sociology, as we11 as for a number of more recent publications (Coleman 1968; Mayer 1972; Bartholomew 1973). Because of a neglect of the representation considerations that are discussed here, serious deficiencies exist with the estimation procedures used in these works. An additional reason for concentrating on compatiblilty with a continuous-time Markov framework is that the conceptual issues which must be addressed with more complicated mathematical structures, such as models of heterogeneous processes, already reveal themselves in this comparatively simple setting.

Representation becomes an issue when we have avallable only Eragmentary data on population movements. Unfortunately, in the study of social phenomena, the common situation is to have very incomplete information about the evolution of an empirical process; frequenty, observations have been taken at only two time points, $t=0$ and $t=t_{1}$, yielding a single transition matrix ${ }^{3} \hat{\mathrm{P}}\left(0, \mathrm{t}_{1}\right)$. What we wish to determine, then, are the conditions which this observed matrix must satisfy for it to be 
viewed as an outcome of a continuous-time Markov model. For matrices satisfying the requisite criteria, we wish further to recover the parameters of the particular Markov structure that underlies the empirfcal process. These issues can be posed most effectively in terms of two sequential considerations--embeddability tests, and the identification problem. In practice, a single calculation is usually informative on both matters.

Embeddability. This issue refers to whether or not an observed transition matrix $\hat{P}\left(t_{1}\right)$ could have arisen via the evolution of a stationary continuous-time Markov process. It is well known that certain stochastic matrices are not compatible with such a formulation; this is the case, for instance, if $\hat{\mathrm{P}}\left(t_{1}\right)$ has an element $\hat{\mathrm{P}}_{i j}\left(t_{1}\right)=0$, but some power of the matrix, say $\hat{\mathrm{P}}\left(t_{1}\right)^{\mathrm{n}}$, has a non-zero entry in the same position, $i . e$. , $\hat{\mathrm{p}}_{1 \mathrm{j}}{ }^{(\mathrm{n})}\left(\mathrm{t}_{1}\right) \neq 0$ (Chung 1967, p. 126). Also, according to Coleman (1964a, p. 179; 1964b, p. 4), a stochastic matrix in which some main diagonal element is less than another entry in its column could not have been generated by a continous-time Markov process. We sha11 show that Coleman's claim is in error. ${ }^{4}$ For the present discussion, however, the essential point is that while it is recognized that certain transition matrices cannot be represented by this mathematical structure, there is confusion over the full scope of the requirements for embeddabllity. Our first task, then, is to devise tests for determining compatibility of an empirically determined matrix with a continuous-time Markov formulation.

Identification. If the embeddability tests are passed, then we are guaranteed that $\hat{P}\left(t_{1}\right)$ could have been generated by at least one continuoustime Markov process. The identification problem refers to the possibility that the matrix could have originated from the operation of more than 
one Markov process. Consequently, our second task is to delineate the conditions under which the solution for the parameters of the Markov model will be unique. Also, for instances in which these conditions are not satisfied, we will require procedures for recovering the several Markov structures that could have produced the observed matrix, and identifying the particular model from this list which should be associated with the data.

Sampling error and data collection design. Overlaying the questions of embeddability and multiple solutions is the issue of sampling error. In most applications, an empirically determined transition matrix $\hat{\mathrm{P}}\left(\mathrm{t}_{1}\right)$ will have been constructed from a population sample. Repeated surveys of the population would produce somewhat different transition arrays, so we would be well advised to Investigate the sensitivity of our estimate of the underlying Markov structure to sampling error. In particular, with respect to the matter of embeddability, we might wish to inquire whether a non-embeddable $\hat{P}\left(t_{1}\right)$ is "within error distance" of some embeddable matrix $\widetilde{P}$. If this is the case, we could choose to carry out an analysis In which Markov methods are employed using the adjusted (embeddable)

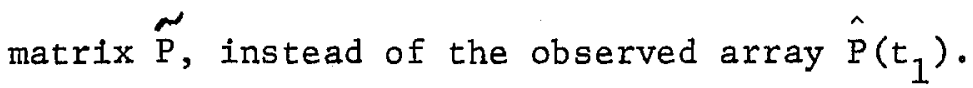

The question of data error leads to more intriguing considerations with respect to the phenomenon of multiple solutions. Even if $\hat{\mathrm{P}}\left(t_{1}\right)$ is compatible with a unique Markov process, it is possible that a slightly modified matrix $\widetilde{P}$--within error distance of the original array-will produce a very different Markov structure from the one that has been 1dentified. As a result, if the data derive from a population sample, then because of sampling variability we may have recovered the wrong Markov structure for the population-level process! We therefore discuss 
strategies for treating an empirically determined matrix as data containing considerable "noise," and identifying from tt the particular Markov model to be associated with the substantive process.

Finally, there are crucial considerations regarding when to survey a population in order to facilitate model identification and parameter estimation. It is widely known, for instance, that if the interval between successive observations is very large (with respect to the rate of evolution of the empirical process), $\hat{P}\left(t_{1}\right)$ will resemble the equilibrium matrix, and the parameters of the continuous-time Markov model which produced the observed array cannot be recovered (Coleman 1968, p. 472). Yet, the Issue of data collection design is considerably more complex than this simple remark conveys, and involves decisions concerning the number of observations to be taken, the spacing between them, and interactions between these considerations.

\section{MATHEMATICAL PRELIMINARIES AND EXAMPLES}

Consider a stochastic process with a finite number of states whose transition probabilities are governed by the system of ordinary differential equations

$$
\frac{d P(t)}{d t}=Q P(t), \quad P(0)=I
$$

where $P(t)$ and $Q$ are $r \times r$ matrices. It is well known (Coleman 1964a, pp. 127-130; Chung 1967, pp. 251-257) that if $Q$ has the structure

$$
q_{i j} \geq 0 \text { for } i \neq j, \quad q_{i 1} \leq 0, \quad \sum_{j=1}^{r} q_{i j}=0 \text { for } i=1, \ldots, r
$$

then the functions $P(t), t>0$, which are solutions of (2.1) comprise the transition-matrices-of continuous-time-stationary-Markov-chains-A-Ey-i-cal- 
element, $p_{i j}(t)$, of $P(t)$ has the interpretation,

$$
\begin{aligned}
p_{i j}(t)= & \text { probability that an individual starting in state } i \\
& \text { at time } 0 \text { will be in state } j \text { at time } t .
\end{aligned}
$$

The Q-arrays, which are known as "Intensity matrices," provide structural information about the population:

(i) $q_{i j} /-q_{i i}=$. probability that an individual in state 1 will move to state $j$, given the occurrence of a transition.

(ii) $1 /-q_{i i}=$ expected length of time for an individual in state $i$ to remain in that state.

We will denote the class of intensity matrices (arrays of the form [2.2]) by the symbol $\underline{Q}$.

Solutions of (2.1) are given by the exponential formula

$$
P(t)=e^{Q t}, \quad t>0
$$

where the matrix exponential $e^{A}$ (A being an arbitrary $r \times r$ matrix) is defined by

$$
e^{A}=\sum_{k=0}^{\infty} \cdot A^{k} / k !
$$

The problem of finding simple test criteria on the entries of an observed stochastic matrix $\hat{P}\left(t_{1}\right), t_{1}<\infty$, which will guarantee that it can be written in the form (2.3) with $Q \varepsilon \varrho$, was first posed by G. Elfving (1937). It has come to be known as the embedding problem for continuoug-time Markov chains.

An obvious description of the subclass $\underset{Z}{Z}$ of stochastic matrices that are embeddable is given by

$$
\underline{\underline{Z}}=\{\mathrm{P} \text { such that } \log \mathrm{P} \in \underline{\underline{Q}}\} \text {. }
$$


Attempts to develop practical test criteria or computer programs to determine membership in $\underline{\underline{Z}}$ are reported in Coleman (1964a, pp. 177-182), Mayer (1972, pp. 327-328), and $\mathrm{Zah} 1$ (1955, p. 97). However, all these investigations suffer from a confusion about the full scope of the embedding problem, as well as from using an incomplete description of the logarthm function of matrix argument. This situation has resulted in a number of erroneous statements about the conditions under which an empirically determined matrix $\hat{\mathrm{P}}\left(\mathrm{t}_{1}\right)$ is, or is not, compatible with a continuous-time Markov process.

Example 1:

Coleman (1964a, p. 179) has asserted that "the most obvious incompatibility is one in which for some state $i, n_{i i} / n_{1}$. is less than some $n_{j i} / n_{j}$. for some state $j . "$ This statemen $t^{5}$ suggests that a Markov structure would not be a suitable model for a large class of mobility matrices (e.g., Prais [1955, table 1]; Coleman [1964a, table 14.8]); Indeed, for any array in which some off-diagonal element exceeds the main diagonal entry in 1ts column. That this assertion is incorrect can be seen from the matrix

$$
\hat{\mathrm{P}}=\left(\begin{array}{llll}
.260 & .169 & .248 & .323 \\
.327 & .275 & .146 & .252 \\
.269 & .346 & .232 & .153 \\
.162 & .285 & .305 & .248
\end{array}\right)
$$

In every column there is a violation of Coleman's necessary criterion, yet this matrix can be represented as $e^{Q}$ with

$$
Q=\left(\begin{array}{rrrr}
-1.700 & .034 & .025 & 1.641 \\
1.573 & -1.657 & .059 & .025 \\
.051 & 1.785 & -1.853 & .017 \\
.017 & .085 & 1.649 & -1.751
\end{array}\right)
$$


Example 2:

E1sewhere, Coleman (1973, p. 21) has written "It is not the case that any discrete-time Markov chain can be generated by an appropriate continuoustime process. Heuristically, those discrete-time chains that cannot be generated by a continuous-time process are those in which the equilibrium distribution is approached through a damped wave, rather than approached asymptotically." 6 Coleman's statement characterizing non-embeddable matrices is incorrect, as the following computations illustrate.

By exponentiating the intensity matrix $Q$ from example $I$ with $t=1$, $P(1.00)=e^{1.00 Q}$, the transition array $(2.4)$ is reproduced. At tIme $t=1.41$,

$$
P(1.41)=e^{1.41 Q}=\left(\begin{array}{llll}
.231 & .233 & .261 & .275 \\
.284 & .244 & .201 & .271 \\
.285 & .296 & .206 & .213 \\
.224 & .299 & .257 & .220
\end{array}\right)
$$

and at time $t=2.24$,

$$
P(2.24)=e^{2.24 Q}=\left(\begin{array}{llll}
.248 & .271 & .239 & .242 \\
.252 & .259 & .235 & .254 \\
.265 & .262 & .223 & .250 \\
.261 & .275 & .226 & .238
\end{array}\right)
$$

Note that each main diagonal entry $p_{i i}(t)$, observed over the three matrices, has the property $p_{i 1}(1.00)>p_{1 i}(1.41)<p_{i 1}(2.24)$. This means that $p_{11}(t)$ approaches an equilibrium value as $t \rightarrow \infty$ through damped oscillations, and not asymptotically. Yet, because of the manner by which the sequence of P-matrices was constructed, they depict the evolution of a continuous-time Markov process.

Example 3:

In attempting to represent an observed matrix $\hat{P}(t)$ in the form (2.3), Zah1 (1955, p. 97) states that "the estimate of $Q$ is taken to be 


$$
\frac{1}{t} \log \hat{P}(t)=\frac{1}{t} \sum_{k=1}^{\infty} \frac{(-1)^{k-1}[\hat{P}(t)-I]^{k}}{k}
$$

provided the series converges." Coleman (1968, p. 472) makes essentially the same claim. Yet, although convergence of (2.5) does provide a representation of $\log \hat{\mathrm{P}}$, it does not guarantee that ${ }^{7} \log \hat{\mathrm{P}} \varepsilon \mathrm{g}$. In particular, consider

$$
\hat{\mathrm{P}}=\left(\begin{array}{lll}
.600 & .330 & .070 \\
.302 & .560 & .138 \\
.380 & .040 & .580
\end{array}\right)
$$

The series representation for $\log \hat{\mathrm{P}}$ converges to

$$
\log \hat{\mathrm{P}}=\left(\begin{array}{rrr}
-.692 & .639 & .053 \\
.496 & -.733 & .237 \\
.707 & -.144 & -.563
\end{array}\right)
$$

which is not in $\underline{Q}$ since $(\log \hat{\mathrm{P}})_{32}=-.144<0$. Example 4:

In possibly the most serious of the misunderstandings, Coleman (1968, p. 472) has asserted that "When [(2.5)] does not converge, this means that the data are not compatible with the assumptions of a continuous-time Markov process, or that the moves of the panel are too widely spaced." Mayer (1972, p. 328) makes essentially the same point: "The fallure of [(2.5)] to converge for all transition matrices $P(t)$ reflects the fact that not all such matrices can arise from a continuous-time stationary Markov chain." These statements are in error. Equation (2.5) may fall to converge for matrices $P$, not resembling the equilibrium matrix, which nonetheless can be represented in the form $e^{Q}$ with $Q \varepsilon Q$. Consider 


$$
\hat{\mathrm{P}}=\left(\begin{array}{lll}
.3654 & .3762 & .2584 \\
.3292 & .3567 & .3141 \\
.4040 & .3188 & .2772
\end{array}\right)
$$

The series representation (2.5) converges if and only if $\left|\lambda_{i}-1\right|<1$ for all eigenvalues $\lambda_{i}$ of $\hat{P}$. The above matrix has eigenvalues $\lambda_{1}=1, \lambda_{2}=.0531$, $\lambda_{3}=-.053 i$. Thus $\left|\lambda_{2}-1\right|=\left|\lambda_{3}-1\right|>1$ and (2.5) diverges. Nevertheless, $\hat{\mathrm{P}}=\mathrm{e}^{\mathrm{Q}}$ for

$$
Q=\left(\begin{array}{rrr}
-1.805 & 1.718 & .087 \\
.044 & -1.784 & 1.740 \\
2.262 & .017 & -2.279
\end{array}\right)
$$

and it is therefore embeddable.

The preceeding examples highlight the confusions that exist concerning which transition matrices can be represented as outcomes of the evolution of a continuous-time Markov process. In particular, we have indicated that the standard recipe for estimating $Q$ (the matrix of structural parameters which govern population movements)--via the power series representation (2.5)--is highly deficient. The series does not provide a complete description of the logarithm of a matrix; as a result, it falls to converge for transition arrays that are compatible with a Markov formulation.

In fact, the inadequacy of equation (2.5) as a procedure for estimatIng the intensity matrix $Q$ is even more fundamental than the above illustrations suggest. While the power series will converge to at most one version of $\log \hat{\mathrm{P}} \varepsilon Q$, the equation $\hat{\mathrm{P}}=e^{Q}$ can have multiple solutions $Q \varepsilon Q$. This is a matter of great importance in sociological investigations because the conventional strategy in using Markov models for theory construction emphasizes decomposing the $q_{i j}$ elements of $Q$ among theoretically postulated effect parameters (Coleman [1964a, chap. 5]; [1964b, chap. 2]; 
McDill and Coleman [1963]). Clearly, one can hardly begin this task without ensuring that the correct $Q$ has been recovered for the substantive process under study. Before considering the issues of multiple solutions and model identification, we address the conceptually prior question of embeddability of $\hat{p}$; that is, we seek to determine which transition matrices are compatible with a continuous-time Markov process.

\section{EMBEDDABILITY OF $\hat{\mathrm{P}}$}

In the case of $2 \times 2$ matrices, a complete and practical solution to the question of embeddability was given by D. G. Kendall (see Kingman [1962], p. 15), who proved that

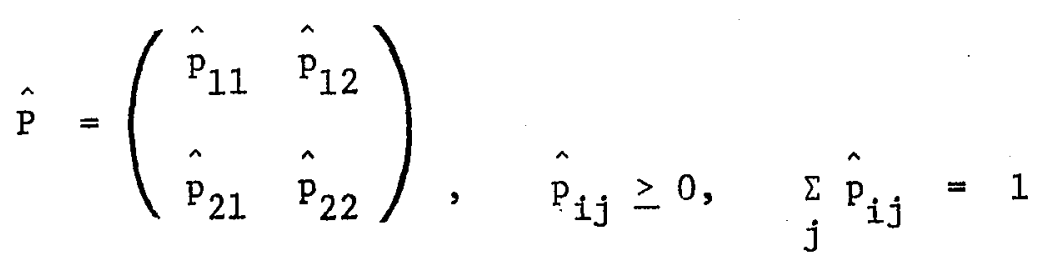

is in $\underline{\underline{Z}}$ (equivalently, can be represented as $e^{Q}, Q \varepsilon Q$ ) if and only if $\hat{\mathrm{p}}_{11}+\hat{\mathrm{p}}_{22}>1$.

A solution to the embedding problem for stochastic matrices with an arbitrary finite number of states was provided by Kingman (1962). In particular, he proved that $\hat{P}$ can be written in the form $e^{Q}$, with $Q \varepsilon \underline{Q}$, if and only if (i) det $\hat{P}>0$, and (ii) for every positive integer $n$, there is a stochastic matrix $P_{n}$ such that $\left(P_{n}\right)^{n}=\hat{P}$. Unfortunately, condition $(11)$ does not lead to practical test procedures to be applied to $\hat{\mathrm{P}}$, and Kingman pointed out the impossibility of obtaining general tests as simple as those in the $2 \times 2$ case for matrices of order greater than or equal to 3 . A further mathematically interesting solution to the embedding problem has recently been given by S. Johansen (1973, p. 180); however, in keeping with Kingman's remarks, it too is not useful for practical computation. 
This impasse has led to the development of a considerable number of easily applicable necessary condttions for an $r \times r$ stochastic matrix $\hat{P}$ to be in $\underline{\underline{Z}}$. These conditions are presented in section 3.1 , with 1llustrations of their use. A common feature of the tests is that they can only be used to assert that a particular matrix is not compatible with a Markov model. An empirically determined matrix which passes all of the tests in section 3.1 must still be subject to an examination based on sufflciency conditions for embeddability, if one hopes to pass on to the stage of model Identification. With the results of Kingman (1962) and Johansen (1973) at hand, our only recourse is to develop simple computational procedures for obtaining all branches of $\log \hat{P}$ compatible with the criteria in section 3.1 , and test these versions of the logarithm for membership in $\underline{Q}$. This seemingly straightforward program leads to some surprisingly subtle phenomena, which are delineated in section 3.2. General practical recommendations for testing an observed matrix $\hat{\mathrm{P}}$ for embeddabilIty are outlined in section 3.3 .

\subsection{Necessary Conditions}

Test criteria which empirically determined matrices must satisfy to be compatible with a family of mathematical models can usefully be viewed as devices for 1solating matrices generated by these models from the class of all stochastic arrays. The necessary conditions listed below are the simplest such tests for distinguishing the subclass of matrices generated by continuous-time Markov models.

Condition 1. (Austin and Ornstein, see Chung [1967, p. 126] for deta11s.) If $\hat{p}_{1 j}\left(t_{1}\right)=0$, then $\hat{p}_{i j}(n)\left(t_{1}\right)=0$ for every integer $n$. If $\hat{p}_{1 j}\left(t_{1}\right) \neq 0$ then $\hat{p}_{1 j}^{(n)}\left(t_{I}\right) \neq 0$ for any integer $n$.

Condition 2. (Kingman 1962) $\operatorname{det} \hat{\mathrm{P}}>0$. 
Condition 3. (E1fving 1937) No eigenvalue $\lambda_{1}$ of $\hat{\mathrm{P}}$ can satisfy $\left|\lambda_{1}\right| \neq 1$ other than $\lambda_{1}=1$. In addition, any negative eigenvalue must have even (a1gebraie) multiplicity.

Condition 4. (Runnenberg 1962) All eigenvalues of $\hat{\mathrm{P}}$ must lie inside a heart-shaped region $\mathrm{H}_{r}$ in the complex plane whose boundary is the curve $x(v)+i y(v)$, where

$$
\left.\begin{array}{l}
x(v)=\left[\exp \left(-v+v \cos \frac{2 \pi}{r}\right)\right] \cos \left(v \sin \frac{2 \pi}{r}\right) \\
y(v)=\left[\exp \left(-v+v \cos \frac{2 \pi}{r}\right)\right] \sin \left(v \sin \frac{2 \pi}{r}\right)
\end{array}\right\}
$$

together with its symmetric image with respect to the real axis. In this parametrized formulation, $r=$ order of the matrix $\hat{P}$, and $v$ is restricted by $0 \leq \mathrm{v} \leq \frac{\pi}{\sin (2 \pi / \mathrm{r})}$. The regions $\mathrm{H}_{3}, \mathrm{H}_{6}$, and $\mathrm{H}_{12}$ are displayed in figures 1,2 , and 3 . The larger cone-shaped zones $\mathrm{K}_{3}, \mathrm{~K}_{6}$, and $\mathrm{K}_{12}$ show the bounds on the elgenvalues of arbitrary $3 \times 3,6 \times 6$, and $12 \times 12$ stochastic matrices.

\section{Figures 1,2 , and 3 about here}

The cone-shaped zones arise from the requirement that the eigenvalues of an arbitrary stochastic matrix $\hat{\mathrm{P}}$ must satisfy ${ }^{8}$

$$
\left(\frac{1}{2}+\frac{1}{r}\right) \pi \leq \arg (\lambda-1) \leq\left(\frac{3}{2}-\frac{1}{r}\right) \pi
$$

(where the argument is in radians), together with the condition $|\lambda| \leq 1$. The additional 1imitation to the heart-shaped set $\mathrm{H}_{\mathrm{r}}$ contained in $\mathrm{K}_{\mathrm{r}}$ arises from the continuous-time Markov assumptions. This restriction can also be described by saying that the eigenvalues of $\hat{P}$ must satisfy (3.2) and

$$
\left(\frac{1}{2}+\frac{1}{r}\right) \pi \leq \arg (\log \lambda) \leq\left(\frac{3}{2}-\frac{1}{r}\right) \pi
$$

Examination of $\mathrm{H}_{3}$ explains why failure of the series (2.5) to converge In 
Figure 1. Llgenvalue Reglong for $3 \times 3$ stochaetlc Matrleo $\left(K_{3}\right)$, and for the Subset of them whlch is in $\underline{z}_{(}\left(1_{3}\right)^{1}$

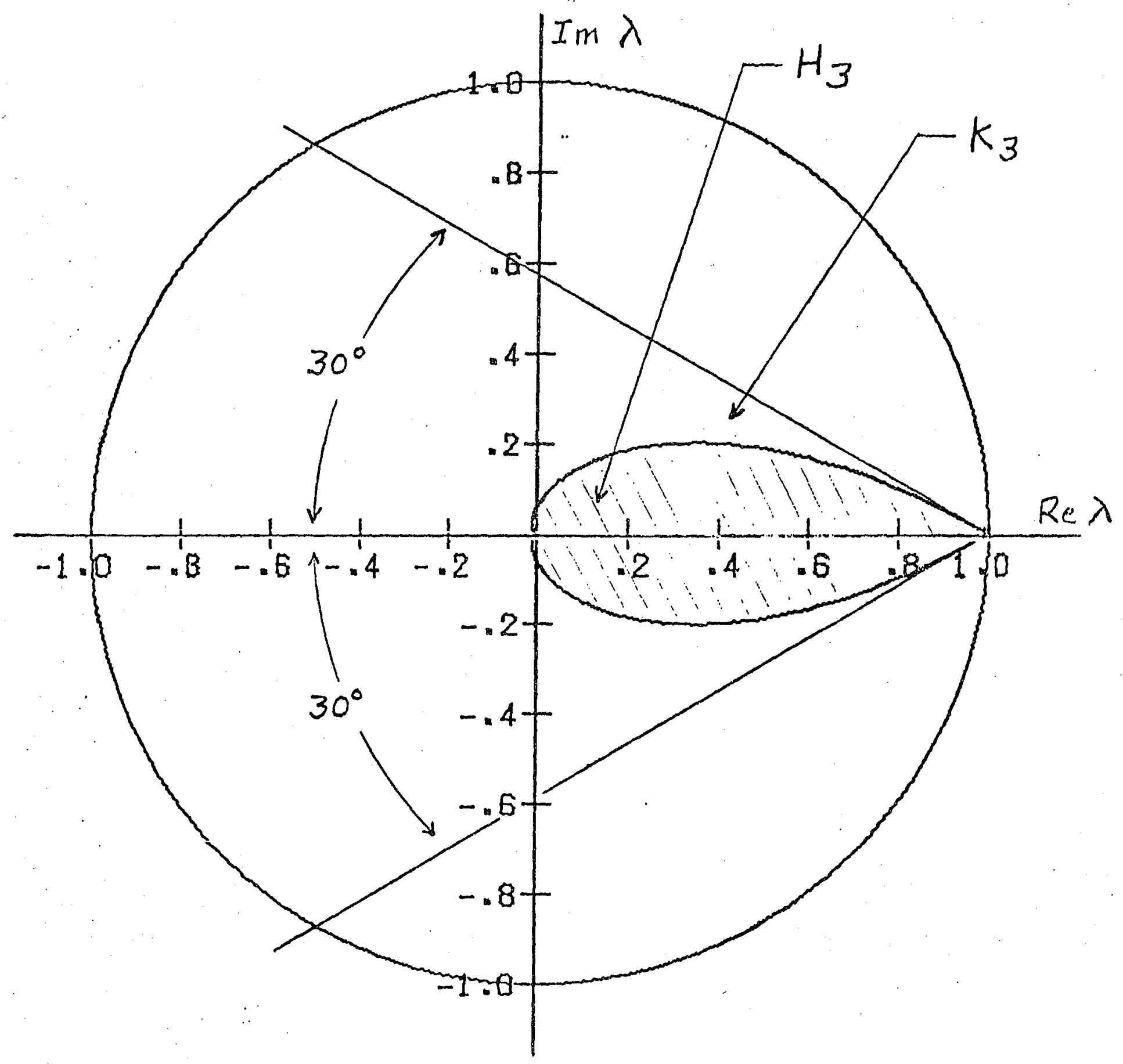

${ }^{A} A$ necosuary condition for $\hat{P}$ to be embediable is that all les olgenvalues lla In the shaded zone. 
Flgure 2. Elgenvalue Reglons for $6 \times 6$ stochastle Matrices $\left(K_{0}\right)$, and for the : Subsot of Them which la in $\underline{\underline{Z}}\left(H_{6}\right)^{1}$

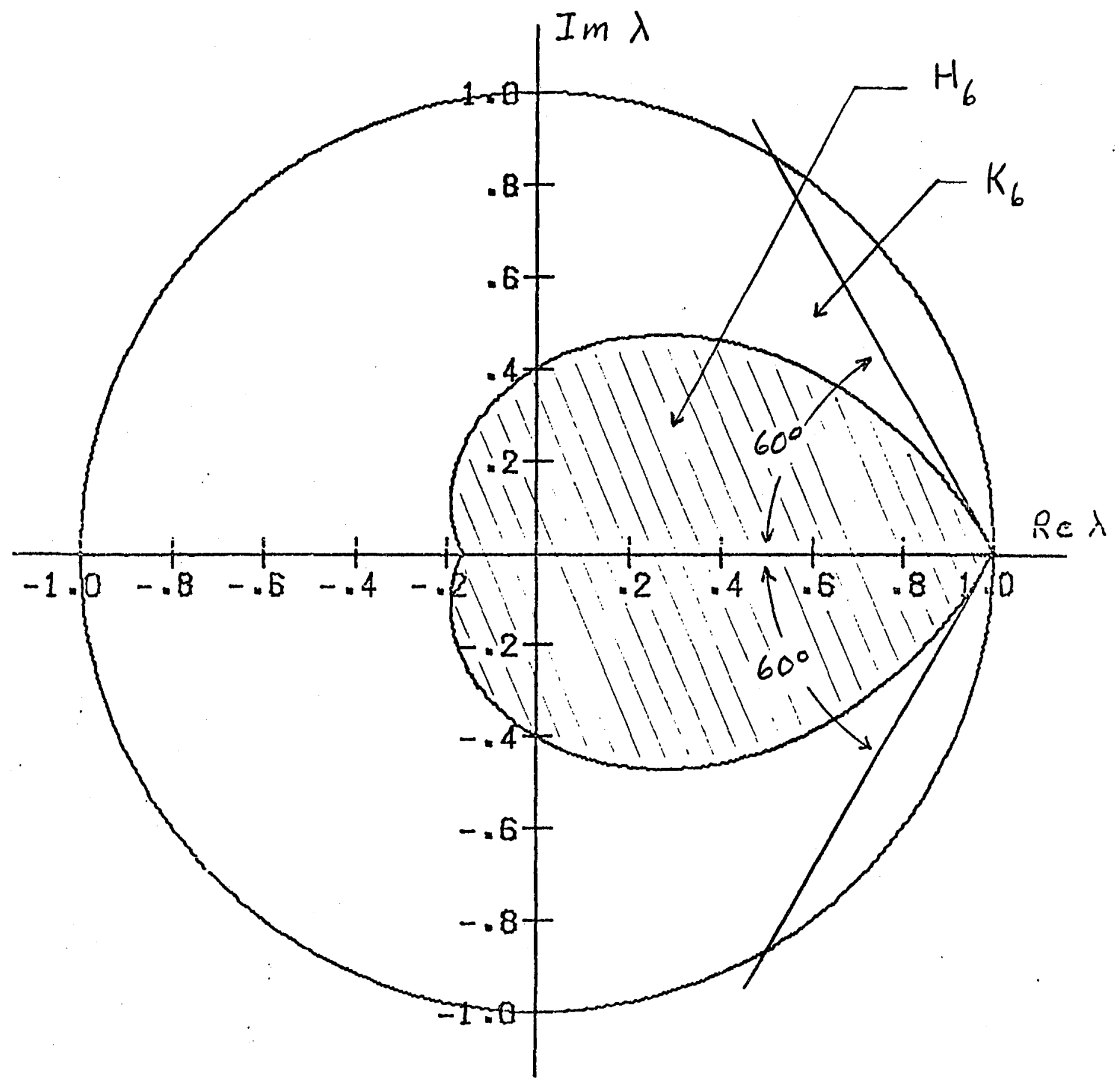

IA necesanzy condition for $\hat{p}$ to be elnbeddablo is that all its olgenvalues lie In the shaded rone. 
Flgure 3. Elgenvalue Keglons for $12 \times 12$ stochaste Matrlces $\left(K_{12}\right)$, and for

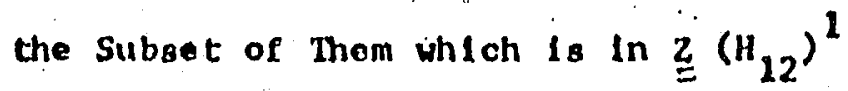

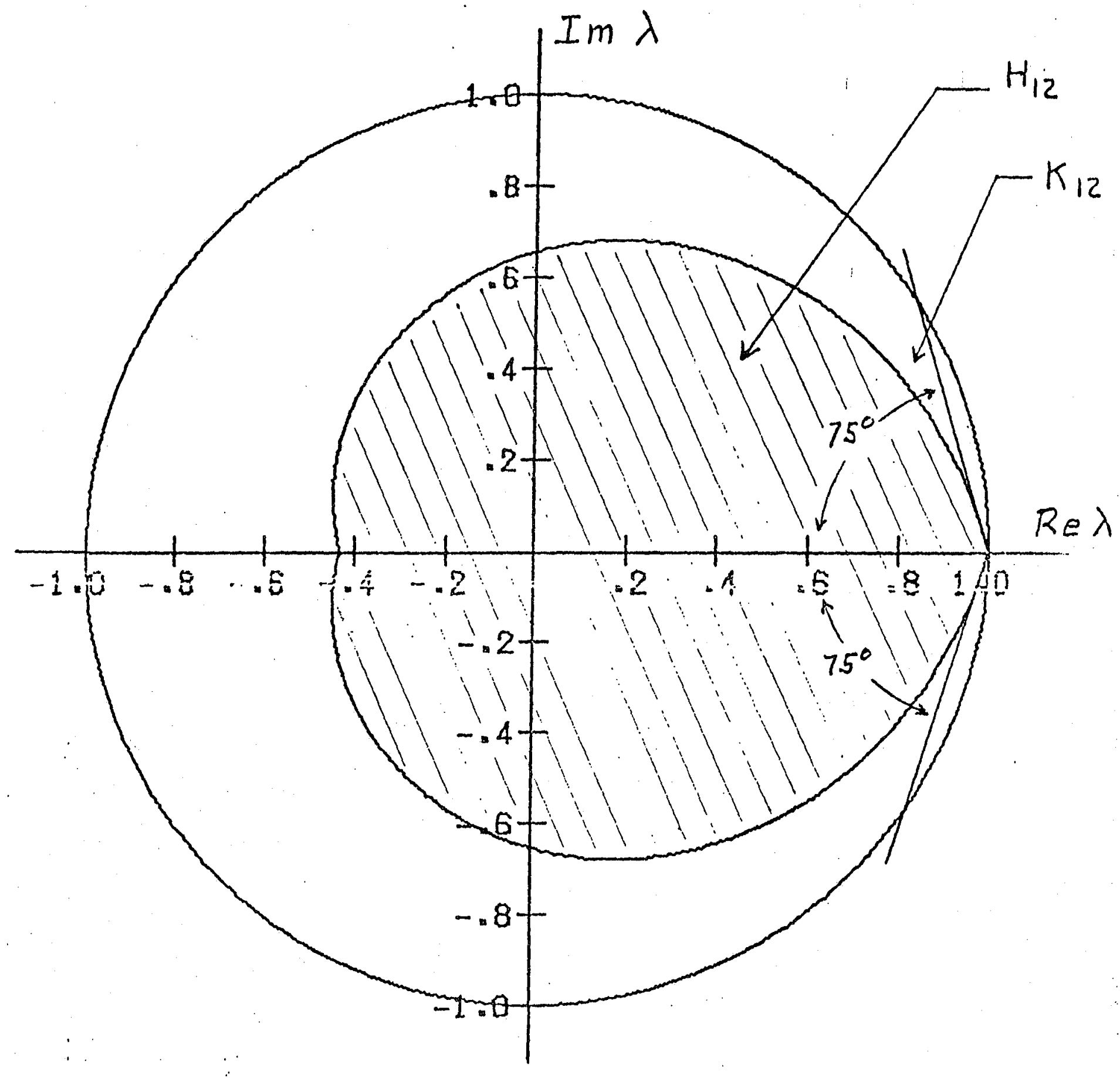

A necessary condltion for $\hat{p}$ to be embeddable is that all ita elgenvalues lle In the shaded rene. 
example 4 did not rule out compatibility of $\hat{P}$ with a continuous-time Markov process. The region of convergence of (2.5) is $\left|\lambda_{i}-1\right|<1$, 1.e., the unit circle centered at $(1,0)$, and the complex eigenvalues of the matrix in that example, while exterior to this region, are inside $\mathrm{H}_{3}$. Example 5:

Suppose you observe the matrix

$$
\hat{\mathrm{P}}=\left(\begin{array}{lll}
.15 & .35 & .50 \\
.37 & .45 & .18 \\
.20 & .60 & .20
\end{array}\right)
$$

Since det $\hat{\mathrm{P}}=.05>0$, condition 2 is satisfied. However, $\hat{\mathrm{P}}$ has eigenvalues $\lambda_{1}=1, \lambda_{2}=-.1+.21, \lambda_{3}=-.1-.21$ which, by (3.2), Ile Inside the cone $\mathrm{K}_{3}$, but they are outside the heart-shaped zone $\mathrm{H}_{3}$. Thus, $\hat{\mathrm{P}}$ cannot be represented as $e^{Q}$ for any $Q \in g$; i.e., it is not compatible with a continuoustime Markov model.

Example 6:

Consider the matrix

$$
\hat{\mathrm{P}}=\left(\begin{array}{lll}
.20 & .40 & .40 \\
.35 & .20 & .45 \\
.40 & .40 & .20
\end{array}\right)
$$

Here, det $\hat{P}=.04>0$, satisfying condition 2 . The elgenvalues of $\hat{\mathrm{P}}$ are $\lambda_{1}=1, \lambda_{2}=\lambda_{3}=-.2$ so that condition 3 applies and is satisfied. Nevertheless, $\lambda_{2}$ and $\lambda_{3}$ are outside the zone $H_{3}$. Thus, $\hat{\mathrm{P}}$ is not compatible with a continuous-time Markov model.

Example 7:

Recall the matrix of example 3 ,

$$
\hat{\mathrm{P}}=\left(\begin{array}{lll}
.600 & .330 & .070 \\
.302 & .560 & .138 \\
.380 & .040 & .580
\end{array}\right)
$$


This matrix satisfies the necessary conditions 1-4; however, it is still not representable as $e^{Q}$ for any $Q \in \underline{Q}$. This assertion is based on an examination of all versions of $\log \hat{P}$ which are candidates for membership in $Q$. An understanding of these tests requires a complete description of $\log \hat{P}$. This is the subject of the next section.

3.2 The Matrix Equation $\hat{P}=e^{Q}$

We require a definition of a function of matrix argument ${ }^{9}$ which is sufficiently general to include analytic functions such as $e^{x}$ and $\log x$. It is useful to motivate the definition by an important property of polynomlal functions $g(x)$. In particular, if

$$
g(x)=a_{0}+a_{1} x+a_{2} x^{2}+\ldots a_{n} x^{n}
$$

and $A$ is an arbitrary square matrix, a natural defintition of $g(A)$ is given by

$$
g(A)=a_{0} I+a_{1} A+a_{2} A^{2}+\cdots+a_{n} A^{n}
$$

In addition, A can always be reduced to Jordan form $J$ by some non-singular matrix $H$, i.e.,

$$
\mathrm{A}=\mathrm{HJH}^{-1}
$$

Finally, it is readily verified that

$$
g(A)=H g(J) H^{-1}
$$

Every Jordan matrix $\mathrm{J}$ has the following block structure:

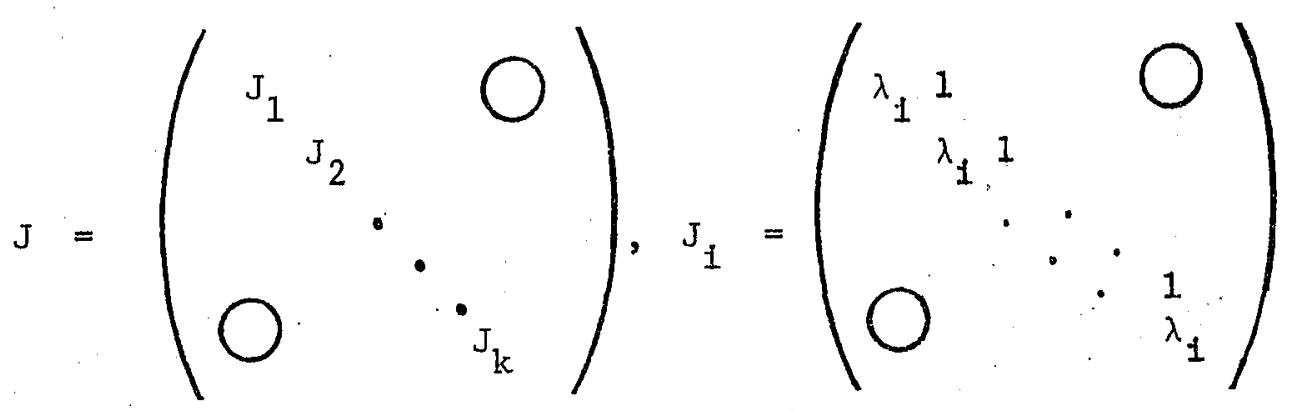


where $\lambda_{i}$ is the $i-t h$ eigenvalue of matrix $A$, and occurs in $J_{1}$ with multiplicity $v_{i}$, the order of $J_{1} \cdot \quad$ (The $\lambda_{i}$ appearing in different blocks $J_{1}$ are $k$

not necessarily distinct.) Also, $\sum_{1} v_{i}=r$, the order of A.

The expression (3.5) will be useful in a wider context than just polynomials provided that we have a representation of $g(J)$ for arbitrary Jordan matrices $\mathrm{J}$, which generalizes to analytic functions ${ }^{10} \mathrm{f}(\mathrm{J})$. Then our program will be to define $f(A)$ according to (3.5), with $g$ replaced by $f$, adding appropriate conventions for multiple-valued functions. For a polynomial function $g(x)$ we introduce its Taylor serles expansion about $x=\lambda_{1}$, and write

$$
g(J)=\left(\begin{array}{ccc}
g\left(J_{1}\right) & & \\
& g\left(J_{2}\right) & \\
& \cdot & \\
& & g\left(J_{k}\right)
\end{array}\right)
$$

where $^{11}$

$$
\begin{aligned}
& g\left(J_{i}\right)=g\left(\lambda_{i}\right) I+g^{\prime}\left(\lambda_{i}\right)\left(J_{i}-\lambda_{i} I\right)+\ldots+\frac{g^{\left(v_{i}-1\right)}\left(\lambda_{i}\right)}{\left(v_{i}-1\right) !}\left(J_{i}-\lambda_{i} I\right)^{v_{1}-1}
\end{aligned}
$$

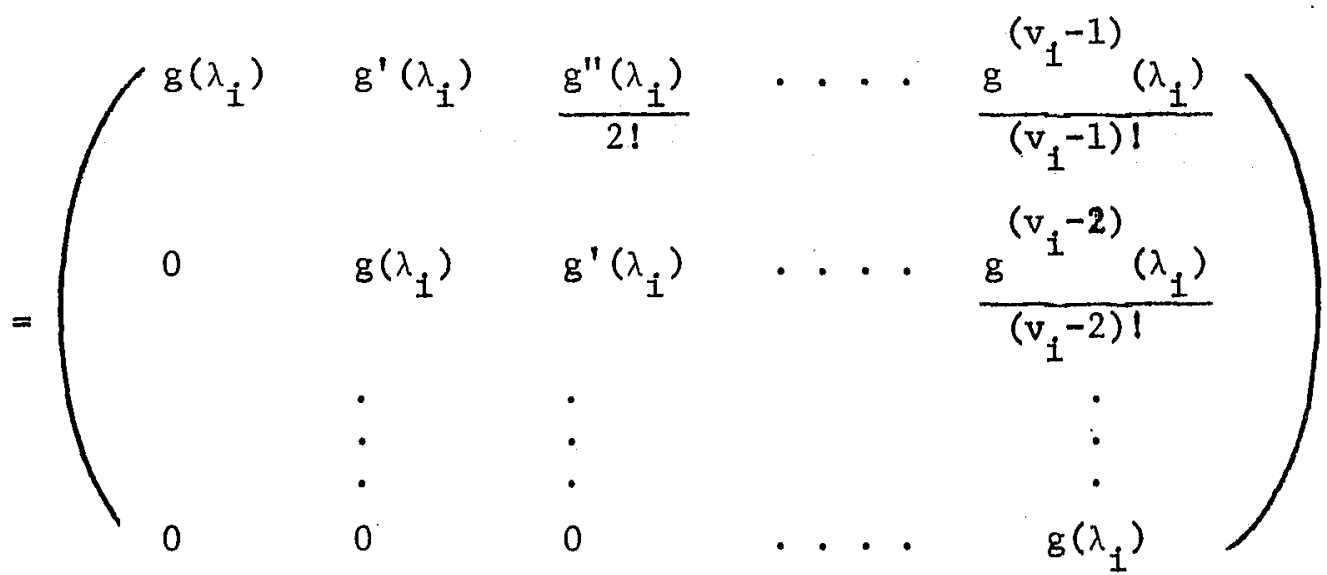


Formula (3.7) has meaning for any function $f$ which is analytic in a neighborhood of the eigenvalue $\lambda_{1}$. Thus, if $f$ is single-valued and analytic in a region of the complex plane contalning the eigenvalues of A (e.g., $f(x)=e^{x}$, we define

$$
f(A)=H f(J) H^{-1}
$$

where $f(J)$ is specified by (3.7) with $g$ replaced by $f$.

If $f$ is multiple-valued (e.g., $f(x)=\sqrt{x}$, or $f(x)=\log x$ ), then we define a branch of $f(A)$ corresponding to the similarity transformation $H$ by

$$
f_{\alpha}(A)=H_{\alpha}(J) H^{-1}
$$

where

$$
f_{\alpha}(J)=\left(\begin{array}{cccc}
f_{\alpha_{1}}\left(J_{1}\right) & & & \\
& f_{\alpha_{2}}\left(J_{2}\right) & & \\
& & & \\
& & & \\
& & & f_{\alpha_{k}\left(J_{k}\right)}
\end{array}\right)
$$

and $f_{\alpha_{i}}(x)$ is any single-valued branch of $f(x)$. Notice that different branches of $f(x)$ may be used with distinct Jordan blocks $J_{1}$, and that each combination of $\left(f_{\alpha_{1}}, f_{\alpha_{2}}, \ldots, f_{\alpha_{k}}\right)$ will generate a different verstion of $f(A)$. Furthermore, the value of $f(A)$ may depend on the chofce of $H$, $a$ point to which we will have cause to return. ${ }^{12}$ Thls definition was introduced by M. Cipolla (1932)--see also, R. F. RInehart (1955)--and represents the necessary level of generality for a discussion of solutions of the matrix equation $e^{Q}=\hat{P}(\hat{P}$ is identified with $A$ in the preceeding discussion). We now specialize to the case where the elgenvalues of $A$ are distinct. The repeated elgenvalue condition, while cruclal to a complete understanding of 
embeddability, is more involved mathematically, and will be consldered separately.

I. Distinct Eigenvalues

In this case, the Jordan matrix $J$ reduces to a diagonal matrix $D$, in which the non-zero entries are the eigenvalues of A. Analogaus to (3.4) we have

$$
\mathrm{A}=\mathrm{HDH}^{-1}
$$

where

$$
D=\left(\begin{array}{ccc}
\lambda_{1} & & \bigcirc \\
\lambda_{2} & & \\
& \cdot & \\
& & \lambda_{r}
\end{array}\right)
$$

Also, the eigenvector corresponding to $\lambda_{i}$ is contained in the 1 -th column of $\mathrm{H}$. The foregoing discussion regarding analytic functions of matrix argument carries over in its entirety, with the functions of Jordan blocks $f\left(J_{1}\right)$ replaced by functions of eigenvalues $f\left(\lambda_{1}\right)$. In particular, when $f$ is multiple-valued, (3.9) reduces to

$$
f_{\alpha}(A)=H f_{\alpha}(D) H^{-1}
$$

where

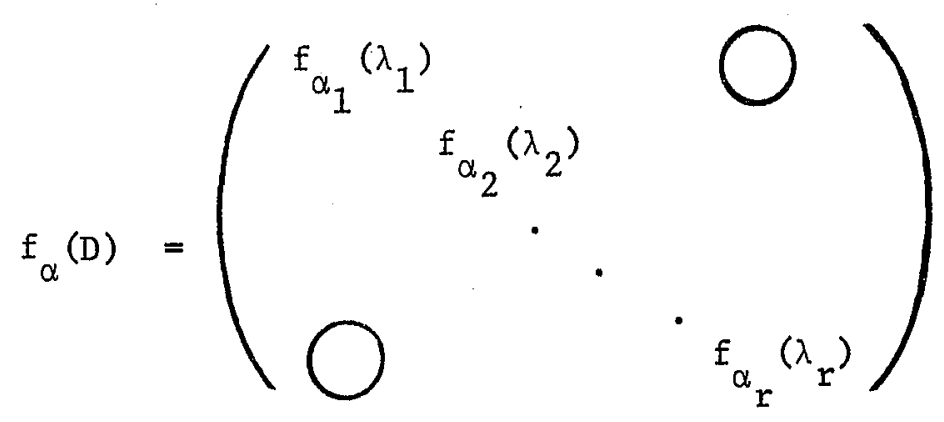


A different version of $f(A)$ is obtained from each combination of branches of $\left(f_{\alpha_{1}}, f_{\alpha_{2}}, \cdots \cdot f_{\alpha_{r}}\right)$.

This discussion is relevant in the following way to the determination of embeddability. Ascertaining compatibility of an observed matrix $\hat{P}$ with a continuous-time Markov process requires investigating whether there exists an array $Q \varepsilon @$ such that $\hat{\mathrm{P}}=\mathrm{e}^{\mathrm{Q}}$. Lacking readily computable sufficiency conditions for general $\mathrm{r} \times \mathrm{r}$ stochastic matrices, our strategy must be to compute $\log \hat{\mathrm{P}}$ and examine $i t$ for membership in $\underline{Q}$. Now, the logarithm function is multiple-valued, 13

$$
\log _{k} z=\log |z|+i(\theta+2 \pi k), \quad k=0, \pm 1, \pm 2, \ldots
$$

where $z$ is an arbitrary complex number, $z=a+b i ;|z|=\sqrt{a^{2}+b^{2}}$; and $\theta=\tan ^{-1} \mathrm{~b} / \mathrm{a}$. Each value of $\mathrm{k}$ generates a different version of $\log \mathrm{z}$, called a branch of the logarithm. In general, an Infinity of branches will exist.

From equations (3.11) and (3.12) we have

$$
\log _{\mathrm{K}} \hat{\mathrm{P}}=\operatorname{Hlog}_{\mathrm{K}} \mathrm{DH}^{-1}
$$

where ${ }^{14}$

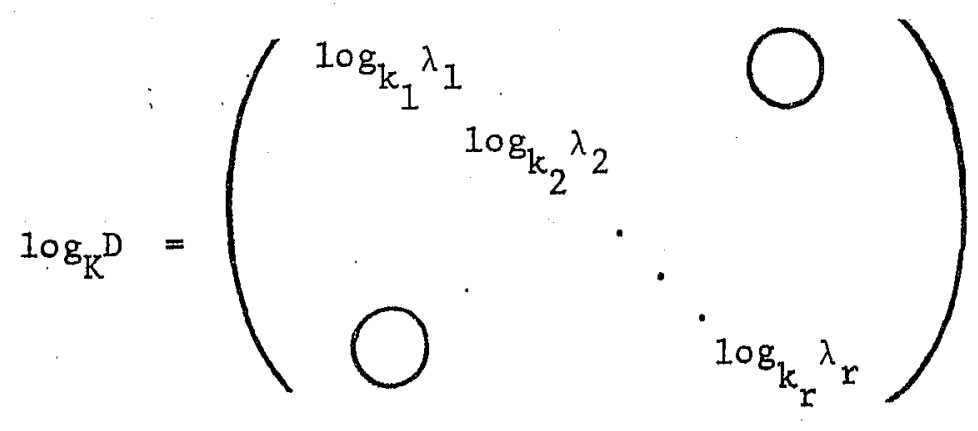

Every combination of values of $\left(\log _{k_{1}} \lambda_{1}, \log _{k_{2}} \lambda_{2}, \ldots, \log _{k_{r}} \lambda_{r}\right)$ In (3.1.3) w111 yield a version of $\log \hat{\mathrm{P}}$, so to determine embeddability one must check

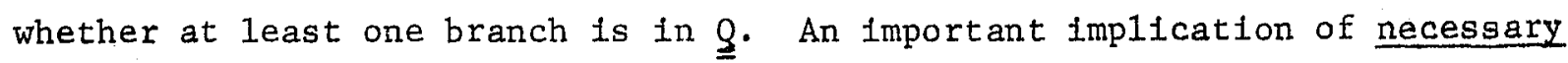


condition 4 in section 3.1 is that only finitely many branches of $\log \hat{\mathrm{P}}$ need be checked for membership in $\underline{Q}$. It is this feature which makes the computational tests, described in detail in section 3.3, feasible. Furthermore, in many applications, the number of branches which must be computed is quite small.

Sylvester's formula. If $\mathrm{A}$ is an $\mathrm{r} \times \mathrm{r}$ matrix with distinct eigenvalues $\lambda_{1}, \lambda_{2}, \ldots, \lambda_{r}$, and if $f$ is single-valued in a nelghborhood of each of the eigenvalues, then equation (3.8) is equivalent to (Sylvester 1883)

$$
f(A)=\sum_{i=1}^{r} f\left(\lambda_{i}\right) \prod_{j \neq i} \frac{\left(A-\lambda_{j} I\right)}{\left(\lambda_{1}-\lambda_{j}\right)}
$$

In addition, if $f$ is multiple-valued, then (3.14), with $f\left(\lambda_{1}\right)$ replaced by $f_{\alpha_{i}}\left(\lambda_{i}\right)$, defines a version of $f(A)$ for each combination of branches of ( $f_{\alpha_{1}}$, $f_{\alpha_{2}}, \ldots, f_{\alpha_{r}}$ ); i.e., this equation is equivalent to (3.11).

Example 8:

Consider the matrix

$$
\hat{\mathrm{P}}=\left(\begin{array}{lll}
.3654 & .3762 & .2584 \\
.3292 & .3567 & .3141 \\
.4040 & .3188 & .2772
\end{array}\right)
$$

which also appeared in example 4 , and identify $\hat{P}$ with $A$ in the above discussion. In order to solve the equation $\hat{\mathrm{P}}=\mathrm{e}^{Q}$, observe that $\hat{\mathrm{P}}$ has distinct efgenvalues $\lambda_{1}=1, \lambda_{2}=.053 i, \lambda_{3}=-.053$. Setting $f(x)=10 g x$ in Sylvester's formula, we obtain

$$
\begin{gathered}
\log \hat{\mathrm{P}}=\log \left(\lambda_{1}\right) \frac{\left.\left(\hat{\mathrm{P}}-\lambda_{2} \mathrm{I}\right) \hat{\mathrm{P}}-\lambda_{3} \mathrm{I}\right)}{\left(\lambda_{1}-\lambda_{2}\right)\left(\lambda_{1}-\lambda_{3}\right)}+\log \left(\lambda_{2}\right) \frac{\left(\hat{\mathrm{P}}-\lambda_{1} I\right)\left(\hat{\mathrm{P}}-\lambda_{3} I\right)}{\left(\lambda_{2}-\lambda_{1}\right)\left(\lambda_{2}-\lambda_{3}\right)} \\
+\log \left(\lambda_{3}\right) \frac{\left(\hat{\mathrm{P}}-\lambda_{1} \mathrm{I}\right)\left(\hat{\mathrm{P}}-\lambda_{2} I\right)}{\left(\lambda_{3}-\lambda_{1}\right)\left(\lambda_{3}-\lambda_{2}\right)}
\end{gathered}
$$




$$
=\left(\begin{array}{rrr}
-1.805 & 1.718 & .087 \\
.044 & -1.784 & 1.740 \\
2.262 & .017 & -2.279
\end{array}\right)
$$

which satisfies criterion (2.2) for membership in $\underline{Q}$. In this calculation we used the principal branches of $\log \lambda_{2}$ and $\log \lambda_{3} ;$ namely, $\log \lambda_{2}=\log (.055)$ $+i \frac{\pi}{2}$, and $\log \lambda_{3}=\log (.055)-i \frac{\pi}{2}$. Any other branch, e.g., $\log \lambda_{2}=\log (.055)$ $+1\left(\frac{\pi}{2}+2 \pi k\right)$ for an integer $k \neq 0$, would yield a version of $\log \hat{\mathrm{P}}$ which 1 s not

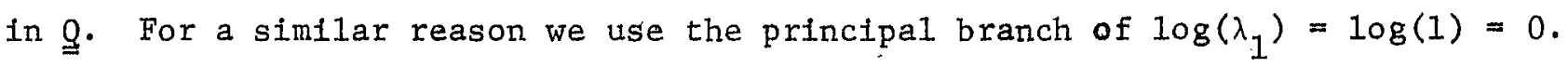
An important feature of this example, and Sylvester's formula in general, is that the logarithm of a matrix is well defined even when the power serles (2.5) diverges, as it does here. For matrices with distinct elgenvalues $\lambda_{1}$ satisfying $\left|\lambda_{i}-1\right|<1$, the series (2.5) is equivalent to the principal branch solution of (3.14)--k =0 in equation (3.12). However, Sylvester's formula is more general in that it will generate all branches of $\log \hat{P}$ as $k$ is varied. ${ }^{15}$ Furthermore, it leads to an evaluation of analytic functions of matrix argument as finite polynomials in the original matrix $\hat{\mathrm{P}}$. The transcendental nature of $f(\hat{P})$ is incorporated entirely in the coeffictents of this polynomial, and involves only functions of elgenvalues. In particular, by rearranging terms, Sylvester's formula for general $x$ x m matrices (3.14) can be written in the form

$$
\hat{f}(\hat{P})=c_{0} I+c_{1} \hat{P}+c_{2} \hat{P}^{2}+\ldots+c_{r-1} \hat{P}^{r-1}
$$

in which the $c_{i}$ 's are scalar functions of the elgenvalues of $\hat{p}$.

\section{Repeated EIgenvalues}

When $\hat{P}$ has one or more sets of equal eigenvalues, the computations to determine embeddability can be considerably more involved. Unfortunately, even though the occurrence of repeated eigenvalues in an observed matrix $\hat{p}$. would be a rare event, we will have reason to consider adjustment strategies 
which make use of this condition. We therefore outline the main issues and analytic procedures at this point; some elaborations are found in section 4.2 and in Appendix 1.

It is useful to categorize matrices with repeated eigenvalues according to whether or not their elementary divisors ${ }^{16}$ are distinct. Distinct elementary divisors means that each eigenvalue $\lambda_{1}$ appears in exactly one Jordan block $J_{i}\left(\lambda_{i}\right)$ in equation (3.6). Non-distinct elementary divisors means that a repeated eigenvalue $\lambda_{1}$ can serve as the diagonal element in more than one Jordan block. The importance of this distinction derives from the fact that the eigenvalues in a block are constrained to be on the same branch of a multiple-valued function--1.e., they must have the same value of $\mathrm{k}$ in expression (3.12). Non-distinct elementary divisors therefore permits different branches of $\log \lambda_{1}$ to be present simultaneously in $\log \mathrm{J}$, via the presence of $\lambda_{i}$ in more than one Jordan block. It is this condition which creates exceptional difficulties in the calculation of $\log \hat{\mathrm{P}}$. The following propositions and examples outline the computations for the two multiple elgenvalue cases:

Proposition 1. If $\mathrm{A}$ is an $\mathrm{r} \times \mathrm{r}$ matrix with $\mathrm{m}$ different eigenvalues $\lambda_{1}, \ldots, \lambda_{\mathrm{m}}$ having. multiplicities $r_{1}, \ldots, r_{\mathrm{m}}$, and elementary divisors $\left(\lambda-\lambda_{1}\right)^{r_{1}}, \ldots,\left(\lambda-\lambda_{m}\right)^{r}{ }_{m}-i . e .$, distinct elementary divisors--and if $f$ is a function that is single-valued and analytic in a nelghborhood of each of the elgenvalues, then $f(A)$ may be computed via (3.8) or by using the equivalent but computationally often simpler formula 17

$$
\begin{aligned}
& f(A)=\sum_{k=1}^{m} \sum_{s=1}^{r_{k}} c_{k s}\left[f\left(\lambda_{k}\right)+\left(A-\lambda_{k} I\right) f^{\prime}\left(\lambda_{k}\right)+\ldots\right. \\
& \left.+\frac{\left(A-\lambda_{k} I\right)^{s-1}}{(s-1) !} f^{(s-1)}\left(\lambda_{k}\right)\right] \underset{j \neq k}{\prod_{j}\left(A-\lambda_{j} I\right)^{r} j\left(A-\lambda_{k} I\right)^{r_{k}-s}}
\end{aligned}
$$


where the terms $c_{k s}$ are the coefficients in the partial fraction expression

$$
\frac{1}{\prod_{k=1}^{m}\left(\lambda-\lambda_{k}\right)}=\sum_{k=1}^{m} \sum_{s=1}^{r_{k}} \frac{c_{k s}}{\left(\lambda-\lambda_{k}\right)^{s}}
$$

When $f$ is multiple-valued, the various branches $f_{\alpha}(A)$ may be found by computing (3.15) for all combinations of branches of $\left(f_{\alpha_{1}}, f_{\alpha_{2}}, \ldots, f_{\alpha_{r}}\right)$ 1.e., $f_{\alpha_{i}}{ }^{(v)}\left(\lambda_{1}\right)$ replaces $f^{(v)}\left(\lambda_{i}\right), v=0,1, \ldots, s-1$, in equation (3.15). With respect to determining embeddability of $\hat{\mathrm{P}}$, the number of versions of $\log \hat{P}=f(\hat{P})$ which need to be examined is discussed in section 3.3 . Example 9:

Consider the matrix

$$
\hat{\mathrm{P}}=\left(\begin{array}{lll}
.1600 & .5300 & .3100 \\
.0527 & .4900 & .4577 \\
.1100 & .1400 & .7500
\end{array}\right)
$$

and identify $\hat{P}$ with $A$ in the preceding discussion. The eigenvalues of $\hat{P}$ are $\lambda_{1}=1$, and $\lambda_{2}=.2$, with multiplicities $r_{1}=1$ and $r_{2}=2$, respectively. First note that both eigenvalues lie in $H_{r}$ (figure 1 ). It is also the case that the elementary divisors of $\hat{P}$ are distinct; they are $(\lambda-1)$ and $(\lambda-.2)^{2}$. We may therefore solve for all solutions to $\hat{\mathrm{P}}=\mathrm{e}^{Q}$ by using equation (3.15) and setting $f\left(\lambda_{1}\right)=\log \lambda_{i}$ :

$$
\begin{aligned}
\log \hat{\mathrm{P}}=c_{11}(\log 1)\left(\hat{\mathrm{P}}-\lambda_{2} I\right)^{2} & +c_{21}\left(\log \lambda_{2}\right)\left(\hat{\mathrm{P}}-\lambda_{1} I\right)\left(\hat{\mathrm{P}}-\lambda_{2} I\right) \\
& +c_{22}\left[\left(\log \lambda_{2}\right) I+\frac{1}{\lambda_{2}}\left(\hat{\mathrm{P}}-\lambda_{2} I\right)\right]\left(\hat{\mathrm{P}}-\lambda_{1} I\right)
\end{aligned}
$$

Selecting the principal branch of the logarithm for each eigenvalue, the first term in expression (3.16) disappears since $\log 1=0$. From the remaining terms, we obtain 


$$
Q=\log \hat{\mathrm{P}}=\left(\begin{array}{rrr}
-2.046 & 1.993 & .053 \\
.024 & -.818 & .794 \\
.315 & .043 & -.358
\end{array}\right)
$$

As in the previous example, we could have chosen some other branch of the logarithm function, $\log \cdot 2 \pm 2 \pi k i$, for an integer $k \neq 0$. However, (3.16) would then produce matrices with complex entries, and these have no meaning in the context of Markov models ( $1 . e .$, they are not in $g$ ).

Proposition 2. All solutions of the equation $e^{Q}=A$ are called branches of the logarithm function of A, and they are given by (Gantmacher 1960, pp. 239-241)

$$
Q=\log A=H B \log \mathrm{J} \mathrm{B}^{-1} \mathrm{H}^{-1}
$$

where

(i) $\mathrm{H}$ is any non-singular matrix which reduces $\mathrm{A}$ to Jordan form, $\mathrm{A}=\mathrm{HJH}^{-1}$.

(ii) $B$ is an arbitrary non-singular matrix that commutes with $\mathrm{J}$, I.e., $B J-J B=0$.

(iii)

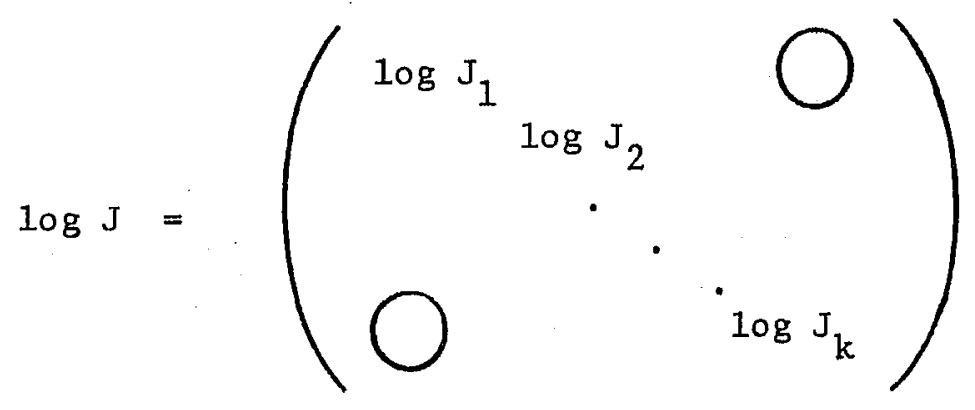

where 


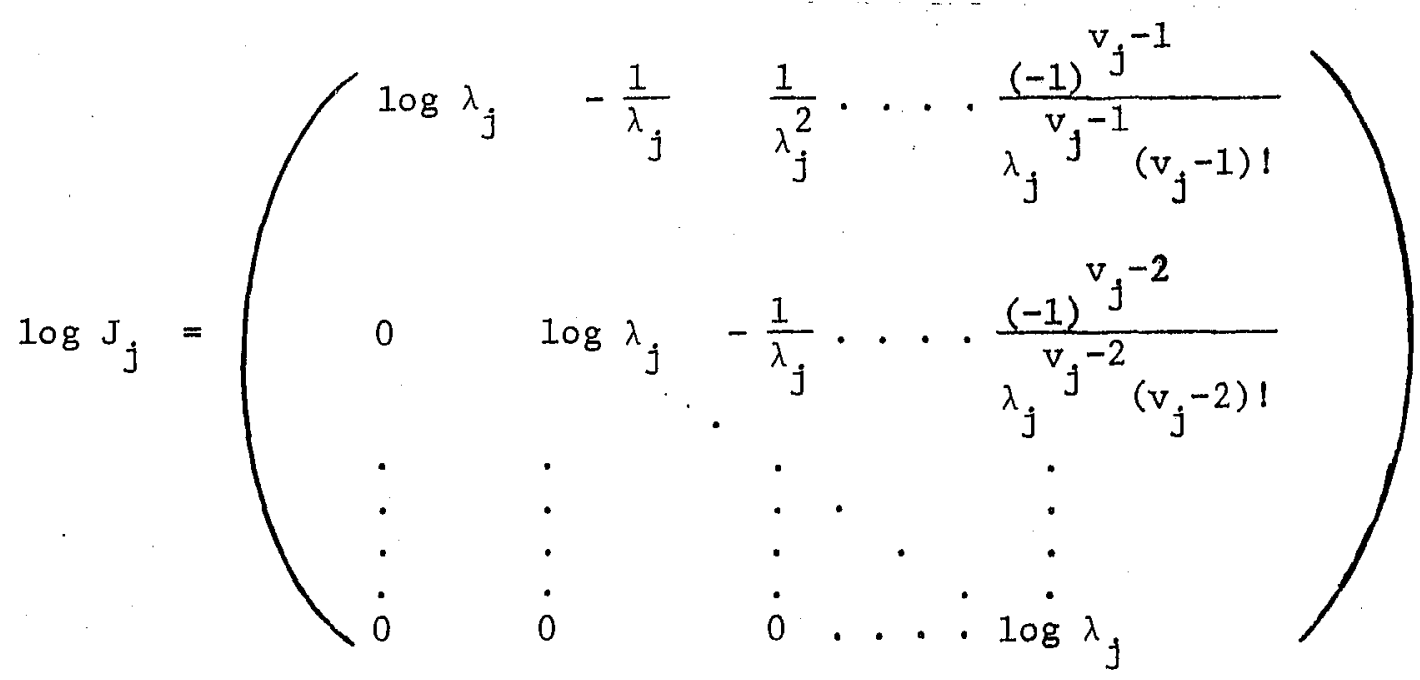

$\log \lambda_{j}=\log \left|\lambda_{j}\right|+i\left(\arg \lambda_{j}+2 \pi k\right), k$ is an integer, and $v_{j}=$ multipl1clty of $\lambda_{j}$ in the elementary divisor $\left(\lambda-\lambda_{j}\right)^{v_{j}}$.

If the elementary divisors of $A$ are distinct, then $B$ may be replaced by the identity in (3.17) and $\log \mathrm{A}$ is independent of the choice of $\mathrm{H}$. It is this property which permits the simpler representations (3.14) and (3.16). When the elementary divisors of $\mathrm{A}$ are non-distinct, computation of all versions of $\log A$ requires a knowledge of the matrices $B$ which satisfy $B J-J B=0$. These matrices contain a finite number of parameters, each of which can be an arbitrary complex number. Every product HB represents a similarity transformation which reduces $A$ to Jordan form and, at the same time, generates a distinct version of $\log A$. This leads to uncountably many versions of $\log \mathrm{A}$, and there may, In fact, be a continuum of such matrices, all or part of which is in $\mathrm{g}$. It is preclsely these matrices with non-distinct elementary divisors which prevent the development of simple general solutions to the embedding problem. In any other situation a researcher need only compute polynomials in $\hat{P}$ to evaluate $\log \hat{P}$, and test a fintie number of branches of the logarithm for membership in $g$. 
Example 10:

Consider the matrix

$$
\hat{\mathrm{P}}=\quad \frac{1}{3}\left(\begin{array}{lll}
1+2 \mathrm{X} & 1-\mathrm{X} & 1-\mathrm{X} \\
1-\mathrm{X} & 1+2 \mathrm{X} & 1-\mathrm{X} \\
1-\mathrm{X} & 1-\mathrm{X} & 1+2 \mathrm{X}
\end{array}\right)
$$

where $X=-e^{-2 \sqrt{3} \pi}$, and identify $\hat{P}$ with $A$ in the preceding discussion. The eigenvalues of $\hat{\mathrm{P}}$ are $\lambda_{1}=1$, and $\lambda_{2}=\lambda_{3}=\mathrm{X}$; the elementary divisors are $(\lambda-1),(\lambda-X),(\lambda-X)$, which are non-distinct. Consequently, the Jordan matrix associated with $\hat{P}$ is

$$
J=\left(\begin{array}{lll}
J_{1} & 0 & 0 \\
0 & J_{2} & 0 \\
0 & 0 & J_{3}
\end{array}\right)=\left(\begin{array}{ccc}
1 & 0 & 0 \\
0 & x & 0 \\
0 & 0 & x
\end{array}\right)
$$

Also, a similarity transformation $\mathrm{H}$ such that $\hat{P}=\mathrm{HJH}^{-1}$ is given by

$$
H=\left(\begin{array}{ccc}
1 & 1 & 1 \\
1 & \frac{1}{2}(-1+i \sqrt{3}) & \frac{1}{2}(-1-i \sqrt{3}) \\
1 & \frac{1}{2}(-1-i \sqrt{3}) & \frac{1}{2}(-1+i \sqrt{3})
\end{array}\right)
$$

In computing $\log \hat{\mathrm{P}}=\mathrm{Q}$ choose $\log \mathrm{J}_{1}=\log 1=0 ; \log \mathrm{J}_{2}=\log \mathrm{X}=$ $-2 \sqrt{3} \pi+1 \pi ;$ and $\log J_{3}=\log x=-2 \sqrt{3} \pi-i \pi$. Now, formula (3.17) with $B=I$, the identity matrix, yields

$$
\log \hat{\mathrm{P}}=2 \pi \sqrt{3}\left(\begin{array}{rrr}
-\frac{2}{3} & \frac{1}{2} & \frac{1}{6} \\
\frac{1}{6} & -\frac{2}{3} & \frac{1}{2} \\
\frac{1}{2} & \frac{1}{6} & -\frac{2}{3}
\end{array}\right)
$$

which belongs to $\mathrm{Q}$. 
To manufacture other versions of $\log \hat{\mathrm{P}}$ which are also in 9 , observe that the matrices which commute with $\mathrm{J}$ are all of the form

$$
B=\left(\begin{array}{ccc}
a & 0 & 0 \\
0 & c_{11} & c_{12} \\
0 & c_{21} & c_{22}
\end{array}\right)
$$

where $\left\{c_{1 j}\right\}$ and a are arbitrary complex numbers subject only to the restriction that $B$ be invertible. For $\log \hat{P}$ to be in $Q$, we may 11 mit consideration to matrices $B$ with entries satisfying,

(1) $c_{11} c_{12}-c_{21} c_{22}=0$,

(11) $u$ is real, where $u=\frac{c_{11} c_{22}+c_{12} c_{21}+2 c_{21} c_{22}}{c_{11} c_{22}-c_{12} c_{21}}$

$$
v \text { is real, where } v=\frac{c_{11} c_{22}+c_{12} c_{21}-2 c_{21} c_{22}}{c_{11} c_{22}-c_{12} c_{21}}
$$

and

$$
\text { (ii1) }|u| \leq 2 \text { and }|v| \leq 2
$$

Conditions (i) and (ii) guarantee that $\log \hat{\mathrm{P}}$ will be real valued, while (11) ensures that the entries will satisfy criterla (2.2). Each choice of $\left\{c_{1 j}\right\}$ then yields a version of $\log \hat{P}$ which is a member of $Q$, and they are all given by

$$
\begin{aligned}
\log \hat{P} & =H B \log \mathrm{J} \mathrm{B}^{-1} \mathrm{H}^{-1} \\
& =2 \pi \sqrt{3}\left(\begin{array}{ccc}
-\frac{2}{3} & \frac{1}{3}+\frac{u}{6} & \frac{1}{3}-\frac{u}{6} \\
\frac{1}{3}-\frac{v}{6} & -\frac{2}{3}-\frac{(u-v)}{12} & \frac{1}{3}+\frac{(u+v)}{12} \\
\frac{1}{3}+\frac{v}{6} & \frac{1}{3}-\frac{(u+v)}{12} & -\frac{2}{3}+\frac{(u-v)}{12}
\end{array}\right)
\end{aligned}
$$

The matrix (3.20) arises in the special case where $c_{11}=c_{22}=1$, 
$c_{12}=c_{21}=0$, and thus $u=v=1$. The non-zero constant a in matrix $B$ does not enter into the formula for $\log \hat{P}$ because it can only multiply the first row of $\log \mathrm{J}$, all of whose entries are 0 .

With this example at hand, ${ }^{18}$ some remarks concerning the role of such matrices in social mobility studies are in order (these comments will be elaborated upon in section 4). If the primary purpose of an investigation Is to obtain structural information about the propensity of individuals In a population to move between particular states, then our major concern must center on the possible values of $q_{i j} /-q_{i j}$ for $i \neq j$ in branches of $\log \hat{P}=Q$ which are in $\underline{Q}$. These ratios have the interpretation, "propensity to move from state $i$ to state $j$ when a change in state occurs." The continuum of branches of $\log \hat{\mathrm{P}}$ which are given by (3.23) represent a continuum of propensities to move between states, all compatible with the observed matrix $\hat{\mathrm{P}}$. Focusing on mobllity out of state 1 in (3.23), we see that

and

$$
0 \leq \frac{q_{12}}{-q_{11}}=\frac{1 / 3+u / 6}{2 / 3} \leq 1
$$

$$
0 \leq \frac{q_{13}}{-q_{11}}=\frac{1 / 3-u / 6}{2 / 3} \leq 1
$$

Thus, on the basis of observations at two time points which glve rise to $\hat{p}$ given by (3.18), we cannot even say whether individuals who start out in state 1 tend to favor state 2 or state 3 when they move. Clearly, this sltuation is totally uninformative about the underlying mobility mechanism, and the present example thereby serves to highlight the unusual difficulties which can arise in the case of repeated eigenvalues with non-distinct elementary divisors. 


\subsection{Summary of Steps to Determine Embeddability}

I. Distinct Eigenvalues

The most common eigenvalue configuration for $\hat{\mathrm{P}}$, an empirically determined stochastic matrix, is one in which the roots are distinct. In testing $\log \hat{\mathrm{P}}$ for membership in $\underline{Q}$, we therefore start with this case.

Step 1. Check that the necessary conditions (1), (2), and (3) In section 3.1 are all satisfied.

Step 2. Check the eigenvalues of $\hat{\mathrm{P}}$ for membership in the heart-shaped zone $\mathrm{H}_{\mathrm{r}}$ described in section 3.1. If this test is passed proceed to steps 3 or 4.

Step 3. If the eigenvalues of $\hat{\mathrm{P}}$ are all real and positive, compute $\log \hat{P}$ using either the power series (2.5), Sylvester's formula (3.14), or the diagonalization transformation (3.11). Only the principal branch of the logarithm ( $k=0$ in equation [3.12]) will be real-valued, and any of the procedures will yield the unique version of $\log \hat{\mathrm{P}}$ that can possibly be in $\underline{Q}$.

Step 4. If $\hat{P}$ has complex eigenvalues they must occur in confugate pairs. For each such pair $(\lambda, \bar{\lambda})$, determine all branches of their logarithms which satisfy: Runnenberg's condition,

$$
\pi\left(\frac{1}{2}+\frac{1}{r}\right) \leq \arg \left(\log _{k} \lambda\right) \leq \pi\left(\frac{3}{2}-\frac{1}{r}\right)
$$

where $r=$ order of matrix $\hat{P}, \arg \left(\log _{k} \lambda\right)=\tan ^{-1}\left(\frac{\theta+2 \pi k}{\log \rho}\right)$, and $k$ specifies a branch of $\log _{k} \lambda$ according to 19

$$
\log _{k} \lambda=\log \rho+i(\theta+2 \pi k) ; \quad k=0, \pm 1, \pm 2, \ldots, 0<\theta<\pi
$$

Now select one of the branches for each complex conjugate pair, and compute 
$\log \hat{\mathrm{P}}$ via (3.11) or by using Sylvester's formula (3.14). Check the resultIng matrix for membership in $\underline{Q}$. Repeat this calculation for all branches satisfying (3.24). Clearly, there are only a finite number of such computations to be performed, and they will yield all versions of $\log \hat{\mathrm{P}} \varepsilon \underline{Q}$.

In particular, if we represent a pair of complex conjugate elgenvalues $(\lambda, \bar{\lambda})$ by $\left(\rho e^{i \theta}, \rho e^{-i \theta}\right), 0<\rho<1$ and $0<\theta<\pi$, then the number of branches of $\log \lambda$ which need to be examined in testing $\log \hat{P}$ for membership in $g$ is $U(r)+L(r)+1$, where

$$
\left.\begin{array}{l}
U(r)=\text { integer part of }\left|\frac{(\log \rho) \tan \left[\pi\left(\frac{1}{2}+\frac{1}{r}\right)\right]-\theta}{2 \pi}\right| \\
L(r)=\text { integer part of }\left|\frac{(\log \rho) \tan \left[\pi\left(\frac{3}{2}-\frac{1}{r}\right)\right]-\theta}{2 \pi}\right|
\end{array}\right\}
$$

and $r$ is the order of the matrix. ${ }^{20} U(r)$ specifies the upper bound to $+k$, and $L(r)$ the lower bound to $-k$, with respect to the multiple-valued logarithm function (3.25). Since the computation of $U(r)$ and $L(r)$ is to be performed for each pair of complex conjugate eigenvalues of $\hat{P}_{\text {; }}$, the number of versions of $\log \hat{P}$ that must be examined is $\prod_{j=1}^{v}\left[U_{j}(r)+L_{j}(r)+1\right]$, where $v$, the upper limit, denotes $\hat{P}$ 's number of complex conjugate eigenvalue pairs. The value of this product will usually be small (frequently $U_{j}(r)=L_{j}(r)=0$ for most j's). In section 4.2 we indicate why it is especially race for a branch other than the principal branch of $\log \hat{\mathrm{P}}$ to be in $\underline{g}$ when the matrix is of low order $(r \leq 3)$. In larger arrays, however, one might have to examine multiple versions of $\log \hat{P}$ to determine embeddability.

II. Data Noise and Repeated Eigenvalues

Because our data are commonly contaminated by the effects of sampling variability and measurement error, one cannot be certain that an empirically determined matrix $\hat{\mathrm{P}}$ is the correct transition matrix for the population of 
interest. As a consequence, if the preceding calculations indicate that $\hat{\mathrm{P}}$ is not embeddable, but the violations in $\log \hat{\mathrm{P}}$ are not severe, a researcher should consider adjusting the observed matrix to a nearby $\tilde{P}$ which is embeddable, and continuing his analysis with the modifled matrix. Strategles for making such an adjustment usually operate on $\log \hat{\mathrm{P}}$, perturbing $1 t$ to a $\operatorname{matrix} Q_{0} \in \underline{Q}$, and then estimate $\widetilde{P}$, the modified array, via $e^{Q_{0}}=\tilde{P}$. There are several procedures for altering $\log \hat{\mathrm{P}}$ so it will satisfy a priori chosen conditions, such as membership in $\underline{Q}$. Zah1 (1955, p. 98) suggests setting the offending elements (negative $q_{1 j}{ }^{\prime} s, 1 \neq j$, in the present context) to zero, and modifying the main diagonal entries so that the row sum condition, $\sum q_{i j}=0$, will be satisfled. Coleman (1964a, pp. 178-180) uses an iterative routine which forces selected $q_{1 f}$ elements to zero in the computation of $\log \hat{\mathrm{P}}$, thereby smearing the compensatory adjustments over the remaining non-zero entries. In example 11, we 11lustrate the adjustment process using yet another procedure, one which minimizes the sum of squared differences between $\log \hat{P}$ and $Q \varepsilon \underline{Q}$. General recommendations regarding which of the techniques is advantageous in a particular problem are currently being prepared.

Example 11:

Suppose you observe the matrix

$$
\hat{\mathrm{P}}=\left(\begin{array}{lll}
.600 & .330 & .070 \\
.302 & .560 & .138 \\
.380 & .040 & .580
\end{array}\right)
$$

which also appeared in example 3 . This matrix has elgenvalues $\lambda_{1}=1$, $\lambda_{2}=.370+.011 i, \lambda_{3}=.370-.011 i$. Applying Runnenberg's condition in the form (3.26) we find that $U=L=0$; hence only the principal branch of 
the logarithm needs to be examined for membership in $\varrho$. Calculating this branch,

$$
\log \hat{P}=\left(\begin{array}{rrr}
-.692 & .639 & .053 \\
.496 & -.733 & .237 \\
.707 & -.144 & -.563
\end{array}\right)
$$

which is not in 9 since $(\log \hat{P})_{32}=-.144<0$. This ralses the question of whether a small perturbation of $\hat{\mathrm{P}}$ would yield a logarlthm in $\mathrm{g}$. To this end we determine the nearest intensity matrix $Q_{0}$ to $\log \hat{P}$, and check whether or not $e^{Q_{0}}$ represents a "small perturbation" of $\hat{P}$. The notion of "nearest" will be defined by $\min _{Q \varepsilon \underline{Q}}|| \log \hat{P}-Q \|$ where ||$A-B||=\sqrt{\sum_{i, j}\left(a_{i j}-b_{i j}\right)^{2}}$. In the present example, the minimum is obtained for

$$
Q_{0}=\left(\begin{array}{ccc}
-.692 & .639 & .053 \\
.496 & -.733 & .237 \\
.635 & 0 & -.635
\end{array}\right)
$$

Calculation of $e^{Q_{0}}=\tilde{P}$ yields

$$
\hat{\mathrm{P}}+(\text { sma11 perturbation })=\tilde{\mathrm{P}}=\left(\begin{array}{lll}
.598 & .334 & .068 \\
.298 & .568 & .134 \\
.349 & .104 & .547
\end{array}\right)
$$

and a case might now be made that $\hat{P}$ was not embeddable only because of sampling error or other data noise. To conclude that the substantive process actually is Markovian with $Q_{0}$ as the governing Intensity matrix, tests of the sort described in section 5 , based on 3 or more time points, must be passed.

Repeated elgenvalues. From a computational point of view, the notion of repeated efgenvalues means that they agree to within a prescribed finfte number of digits. If you take a large random sample of stochastic matrices, then those matrices with repeated eigenvalues tend to occur with a frequency 
close to zero. On the other hand, the entries in $\hat{P}$ which arise in mobllity Investigations are often subject to considerable sampling varlability and other sources of error. Our concern, therefore, is in knowing whether a small perturbation in $\hat{P}$, call it $\widetilde{P}$, would lead to branches of $\log \widetilde{P}$ which are radically different from those of $\log \hat{\mathrm{P}}$. These radical differences can occur in passing from a distinct to a repeated eigenvalue matrix, which in turn can be viewed as being "within error distance" of the original distinct eigenvalue matrix. This suggests that a distinct elgenvalue matrix $\hat{P}$ which is compatible with a Markov model and which has a pair of elgenvalues within a prescribed number of digits of each other, should be perturbed to a $\widetilde{P}$ with repeated eigenvalues. Then the structure of the continum should be displayed as in example 10. If the branches of $108 \widetilde{\mathrm{P}}$ which are in $g$ are sufflciently varied, this would lead us to report that our observations $\hat{p}$ based on data collected at two time points are uninformative about the underlying mobility mechanism.

The additional tasks to be undertaken, then, in a situation where $\hat{p}$ has eigenvalues which are close to being repeated consist of carrying out the following procedures:

Step. 5. Adjust the observed stochastic matrix $\hat{\mathrm{P}}$ so that $1 \mathrm{t}$ will have repeated eigenvalues.

Step 6. Determine the structure of the continuum using the simulation strategy described in Appendix 1, and check whether some part of the continuum is in $\mathrm{Q}$.

The task of adjusting $\hat{P}$ so it will have repeated eigenvalues is not difficult in the case where the elgenvalues close together are complex conjugates. Fortunately, it is this situation which is of primary practical 
interest. If we represent these eigenvalues in polar forn, $(\lambda, \bar{\lambda})=\left(p e^{\perp \theta}\right.$, $\left.\rho e^{-1 \theta}\right), 0<\theta<\pi$, where $\theta \approx 0$ or $\theta \approx \pi$, then the corresponding eigenvalues in $\log \hat{\mathrm{P}}$ are $\log \rho \pm i(\theta+2 \pi k)$. We now to alter $\hat{p}$ so that one of the approximate equalities is replaced by an exact equality. For a scalar $t$, $t \log \hat{P}=H t \log D^{-1}$ will have among its elgenvalues $t \log \rho \pm 1(t \theta+2 \pi k t)$. Therefore, if we choose $t=t_{1}=\frac{2 \pi k}{2 \pi k+\theta}$ or $t=t_{2}=\frac{\pi+2 \pi k}{\theta+2 \pi k}$, where $k$ is the largest branch number $2 \dot{I}$ that satisfies (3.24), the matrix $\tilde{P} \cdot e^{t} \log \hat{P}$ will hảve rểpeated reàl eigenvalues, either $\left(\rho^{t_{1}} e^{12 \pi k}, \rho{ }^{t} e^{-i 2 \pi k}\right)=\left(\rho^{t}\right.$, $\left.\rho^{t} 1\right)$ or $\left(\rho^{t} e^{i \pi(2 k+1)}, \rho^{t} 2 e^{-i \pi(2 k+1)}\right)=\left(-\rho^{t} 2,-\rho^{t}\right)$. This technique is called "riding $\log \hat{p} . "$. It was employed in example 2, and it is applied again in section 4.2 .

\section{MULTIPLE SOLUTIONS OF $\hat{P}=e^{Q}$}

\subsection{Conceptual Overview}

The tests outlined in the preceding section permit a researcher to ascertain whether or not an empirically determined matrix $\hat{P}\left(t_{1}\right)$, constructed from observations at times $t=0$ and $t=t_{1}$, is compatible with a continuoustime Markov process. When the answer is affirmative, at least one version of $\log \hat{\mathrm{P}}\left(\mathrm{t}_{1}\right)$ will be in $\mathrm{g}$. In general, as we have observed, $1 \mathrm{t}$ may be necessary to examine several branches of $\log \hat{\mathrm{P}}\left(t_{1}\right)$ to resolve the question of embeddability. For instance, when $\hat{P}\left(t_{1}\right)$ has complex eigenvalues, each complex conjugate pair will generate $U+L+1$ candidates for membership in $\underline{\underline{s}}$.

In discussing the tests in section 3 our objective was to Investigate embeddability; we sought to determine whether any of the $\log \hat{P}\left(t_{1}\right)$ cand1dates was, in fact, a bonafide member of $g$. In the present section, we shift emphasis and inquire into how many versions of $\log \hat{P}\left(t_{1}\right)$ can belong 


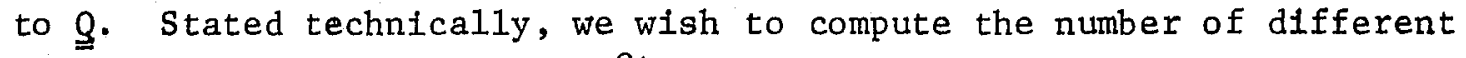
solutions $Q$ to the equation $e^{Q t_{1}}=\hat{P}\left(t_{1}\right)$ which have the required structure (2.2). In the discussion that follows, we shall assume $\hat{P}\left(t_{I}\right)$ is embeddable; 1.e., at least one version of the logarithm is in $Q$.

Under certain conditions it is possible to guarantee that this solution $Q \varepsilon Q$ will be unique. In particular, this is the case whenever one of the following sufficiency conditions is satisfled:

(1) The eigenvalues of $\hat{P}\left(t_{1}\right)$ are distinct, real, and positive.

(11) $\min _{i}\left\{\hat{p}_{i 1}\left(t_{1}\right)\right\}>\frac{1}{2}$, where $\hat{p}_{i 1}\left(t_{1}\right)$ is the diagonal element in the 1-th row of $\hat{P}\left(t_{1}\right)$.

(iii) $\operatorname{det} \hat{\mathrm{P}}\left(t_{1}\right)>\mathrm{e}^{-\pi}=.0432$

The first criterion derives from the fact that only the principal branch of $\log \hat{P}\left(t_{1}\right)$ is real-valued under the indicated eigenvalue constraints. A1so, in this circumstance, the assessment that $Q=\frac{1}{t_{1}} \log \hat{P}\left(t_{1}\right) \varepsilon Q$ wi11 be unique is independent of the cholce of $t_{1}$, since the eigenvalues of $P(t)$ generated by such a $Q$ retain the specified propertles for all times $t$. Additionally, when the eigenvalues satisfy (1), the series formula (2.5) will converge to the unique version of the logartthm in $\mathrm{g}$.

The second and third criterla were established by J. Cuthbert (1972; . 1973), and refer to the specific times $t$ in the evolution of $P(t)=e^{Q t}$ at which the solution $Q \varepsilon Q$ will be unique. For the purpose of model 1dentif1cation, conditions (i1) and (iil) reveal that every Markov chain (1dentifled by a matrix $Q \varepsilon Q$ via the relation $P(t)=e^{Q t}$ ) has an interval of time $[0, T]$ during which only one version of $\log P\left(t_{1}\right), 0<t_{1}<T$, is in $Q$. [The location of the uniqueness interval at the origin follows from the 
fact that $L(t)$, the number of branches of $\log P(t)$ in $Q$, is a non-decreasing function of time--see figure 6 , section $4.2,1$

These comments suggest that in planning an observational study where Markov models are to be utilized for identifying non-directly observable mobility mechanisms (Q-matrices), it is advisable to take the first two observations as close together as possible, while still allowing a representative amount of movement to occur. The question of what constitutes an appropriate time Interval is clearly tied to the nature of the particular substantive process. The point to be highlighted here is that because of the complications which arise when there are multiple solutions, this sort of consideration is consequential in developing sampling strategies for situations where the number of time points at which data can be collected is very restricted.

Except when one of the special conditions (i), (ii), or (iii) is satisfied, it is possible for several branches of $\log \hat{P}\left(t_{1}\right)$ to be in $Q$. This non-uniqueness phenomenon, illustrated in the examples below, has received very little attention in scientific disciplines (physics, engineering, sociology) In which Markov processes are frequently utilized. Nonetheless, the existence of multiple solutions $Q \varepsilon \underline{Q}$ to the equation $e^{Q t_{1}}=\hat{P}\left(t_{1}\right)$ Is not at all uncommon.

Example 12:

Consider the empirically determined matrix

$$
\hat{\mathrm{P}}\left(t_{1}\right)=\left(\begin{array}{llll}
.234 & .252 & .264 & .250 \\
.252 & .237 & .245 & .266 \\
.268 & .255 & .230 & .247 \\
.248 & .271 & .248 & .233
\end{array}\right)
$$


This array can be represented in the form $e^{Q t_{1}}, Q \varepsilon Q$, with either of the matrices

$$
Q_{1}=\left(\begin{array}{rrrr}
-3.350 & .134 & .067 & 3.149 \\
3.132 & -3.306 & .144 & .030 \\
.035 & 3.233 & -3.395 & .127 \\
.137 & .033 & 3.149 & -3.319
\end{array}\right)
$$

or

$$
Q_{2}=\left(\begin{array}{rrrr}
-3.329 & 3.312 & .005 & .012 \\
.033 & -3.337 & 3.209 & .095 \\
.016 & .023 & -3.334 & 3.295 \\
3.294 & .050 & .027 & -3.371
\end{array}\right)
$$

From the perspective of uncovering structural mechanisms, the matter of Identifying the "correct" $Q$ for an empirical process must be a central conslderation because the alternative intensity matrices consistent with the mathematical formalism $\hat{P}\left(t_{1}\right)=e^{Q t_{1}}$ will lead to different substantive conclusions. If only the branch $\frac{1}{t_{1}} \log \hat{P}\left(t_{1}\right)=Q_{1}$ were recovered, one would assert that the most frequent transitions are $s_{1}+s_{4}, s_{2}+s_{1}, s_{3} \rightarrow s_{2}$, and $S_{4}+S_{3^{\circ}}$ In contrast, if only the branch $\frac{1}{t_{1}} \log \hat{P}\left(t_{1}\right)=Q_{2}$ were computed, one would contend that the process evolves principally through the following pattern of movements: $s_{1} \rightarrow s_{2}, s_{2}+s_{3}, s_{3}+s_{4}$, and $s_{4}+s_{1}$. Since in applications of continuous-time Markov processes attention has been directed to the relative magnitudes of the $q_{i j}$ entries, and to apportioning these elements among theoretically specified effect parameters (e.g., Coleman 1964a, chap. 6; McD111 and Coleman 1963; Bartholomew 1973, chap. 5), 1dent1fication of the appropriate intensity matrix would appear to be a necessary Initial step in this sort of analysis. 
This task may. be divided into two component issues: (a) recovery of all matrices $Q \varepsilon \underline{\underline{Q}}$ that are compatible with the representation $e^{Q t_{1}}=\hat{P}\left(t_{1}\right)$, and (b) selection from this 1ist of alternative Q-matrices the correct one for the empirical process at hand. Procedures for accomplishing the first task are presented in the current section. The second issue can be resolved either by bringing additional substantive Information to bear on the nature of the process to ald in choosing among the alternative Q-matrices, or by collecting data at more than two time points, or by sampling the population over a briefer time interval (e.g., within the region of uniqueness). These matters will be considered in section 5 .

4.2 How Multiple Verstons of $\log \hat{\mathrm{P}}(t) \varepsilon Q$ Arise

The simplest way to describe how multiple matrices $Q \varepsilon Q$ originate is to consider the case of a general $3 \times 3$ stochastic matrlx $P(t)$ which has complex elgenvalues. Expressing this matrix in diagonal form we have, $P(t)=\mathbb{D D}(t) \mathrm{H}^{-1}$. For convenience we write the complex igenvalues of $:$ $P(t)$ as exponentials,

$$
D(t)=\left(\begin{array}{ccc}
1 & 0 & 0 \\
0 & \lambda(t) & 0 \\
0 & 0 & \overline{\lambda(t)}
\end{array}\right)=\left(\begin{array}{ccc}
1 & 0 & 0 \\
0 & e^{t(a+b 1)} & 0 \\
0 & 0 & e^{t(a-b i)}
\end{array}\right)
$$

where $\bar{\lambda}(t)$ denotes the complex conjugate of the efgenvalue, $\lambda(t)$. Then, $\log \hat{P}(t)=\operatorname{Iog} D(t) \cdot H^{-1}$, in which

$$
\log D(t)=\left(\begin{array}{ccc}
0 & 0 & 0 \\
0 & a t+i(b t+2 \pi k) & 0 \\
0 & 0 & a t-i(b t+2 \pi k)
\end{array}\right), \quad k=0, \pm 1, \pm 2, \ldots
$$

We specify $b>0$. Also note, for reference, that because $|\lambda(t)|<1$ for al1 $t$, at $=\log |\lambda(t)|<0$. 
Applying Runnenberg's necessary condition for embeddability (3.24), we have

$$
\frac{5 \pi}{6} \leq \tan ^{-1}\left(\frac{b t+2 \pi k}{a t}\right) \leq \frac{7 \pi}{6}, \quad k=0, \pm 1, \pm 2, \ldots
$$

where the inverse tangent specifies arg $(\log \lambda(t))$ in (3.24). For a flxed $t$, we therefore have a series of tests, one for each integer (branch) $k$. The point to be emphasized here is that since every branch of $\log P(t)$ whose eigenvalues satisfy (4.3) is a candidate for membership in $\varrho$, more than one version of the logarithm may, in fact, be in $g$. It is also the case that as $t$ increases, and $P(t)$ evolves to the equilibrium matrix of the process, the number of branches of $\log P(t)$ that are potentially in $g$ becomes larger. These phenomena are illustrated in figures 4 and 5 .

\section{Figures 4 and 5 about here}

Figure 4 displays the locations of various branches 22 of the eigenvalues $\log \lambda(t)=(a \pm b i) t+2 \pi k, t=1$, in relation to Runnenberg's criterion. The wedge-shaped region (solid lines) defines the boundaries of this necessary condition for embeddability--all eigenvalues of $\log P(t)$ must lie in the zone. In this 11lustration, only the principal branch $(k=0)$ Is located in the wedge-shaped region; other branches of the logarithm, which differ by multiples of $2 \pi$ in their Imaginary parts, lie outalde the wedge.

Now consider the effect of letting $t$ increase. With respect to the principal branch of $\log \lambda(t), \tan ^{-1}\left(\frac{b t}{a t}\right)=\tan ^{-1}(b / a)$ and hence the argument of the logarithm is unchanged. With regard to any other branch $k>0$, since

$$
\frac{b t+2 \pi k}{a t}=\frac{b+2 \pi k / t}{a}>\frac{b+2 \pi k}{a}
$$


Figure 4. Elgenvalues of $\log P(t)$, for $t=1$

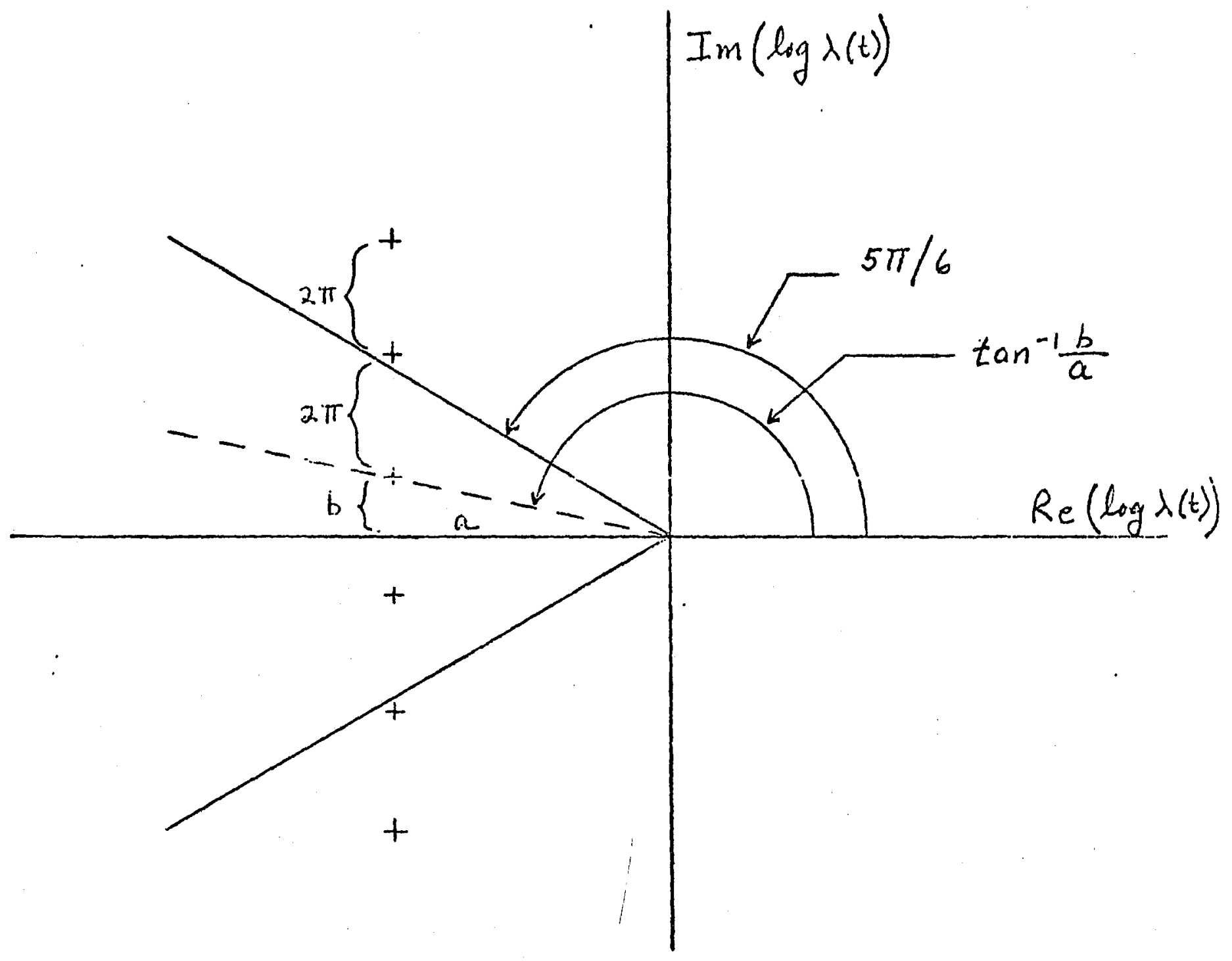

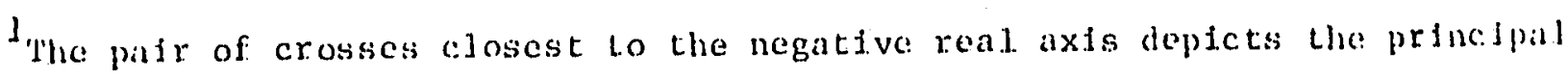
branch of the logarithm, $\log \lambda=a \pm b i$. The pafr next further out represcents the branch for $k=1,1, c, \log \lambda=a \pm f(b+2 \pi)$. And so forth. 
Figure 5. Trajectories of the Eigenvalues of $\log P(t)$, as a function of $L$ ime'

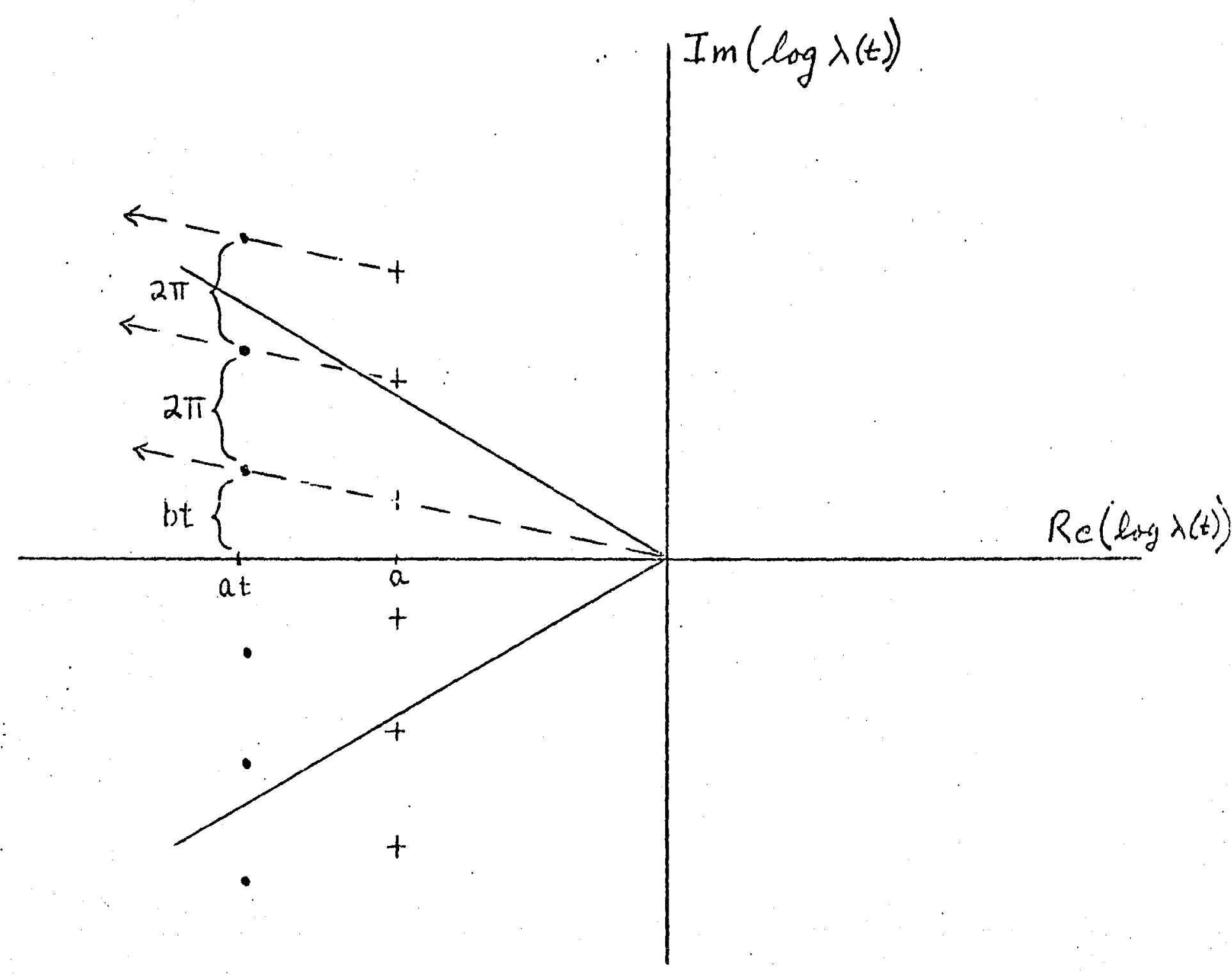

Ihe dashed 1 ines weth arrowheads show the trajectordes of the branchus of $\log \lambda(t)$, as a function of $t$. 
(the inequality follows because $a<0$ ), and since $\tan ^{-1} x$ is an increasing function of $x$ in the second quadrant, we have

$$
\begin{aligned}
\arg (a t & +i[b t+2 \pi k])=\tan ^{-1}([b t+2 \pi k] / a t) \\
& >\tan ^{-1}([b+2 \pi k] / a)=\arg (a+1[b+2 \pi k])
\end{aligned}
$$

This calculation shows that the angle made by a branch of the logarithm $(k>0)$, with respect to the positive real axis, enlarges with time. As a result, additional branches enter the wedge, and the number of versions of $\log P(t)$ that are candidates for membership in $\mathrm{Q}$ increases. This phenomenon is illustrated in figure 5 .

If we let $L(t)=$ \{number of branches of $\log P(t) \varepsilon g \mid$ given $t$, the next relevant considerations are: (i) $L(t)$, itself, is a monotone non-decreasing function of $t$ (except, possibly, for isolated time points), and (11) If $L(t)>1$ at some time $t$ (other than one of the isolated time points), then $L(t) \rightarrow \infty$ as $t \rightarrow \infty$ (Cuthbert 1972; 1973). The graph of $L(t)$ in figure 6 is the prototype for the evolution of any Markov chain where Q has distinct. eigenvalues and at least one complex conjugate pair. At times $t=\pi / b$, $2 \pi / b, 3 \pi / b, \ldots, n \pi / b, \ldots$, the complex conjugate elgenvalues of $P(t)$ w1Il equal $\exp [a n \pi / b \pm 1(n \pi+2 \pi k)], n=1,2,3, \ldots$ and $k=0, \pm 1, \pm 2, \ldots$. This expression reduces to one of the multiple real root conditions, either $\lambda_{2}(t)=\lambda_{3}(t)=\exp (\operatorname{an} \pi / b)$ or $\lambda_{2}(t)=\lambda_{3}(t)=-\exp (\operatorname{an} \pi / b)$, according to whether $\mathrm{n}$ is even or odd. The point to be stressed is that at these times $P(t)=e^{Q t}$ has repeated eigenvalues with non-distinct elementary divisors, which will give rise to a continuum of branches of $\log P(n \pi / b)$.

\section{E1gure 6 about here}

From the point of view of model identification--determining the correct $Q \varepsilon Q$ for a substantive process--these times are a source of difficulty 
47

Figure 6. Number of Branches of $1.0 \mathrm{~g} P(t)$ In $\mathrm{Q}$, as a function or the

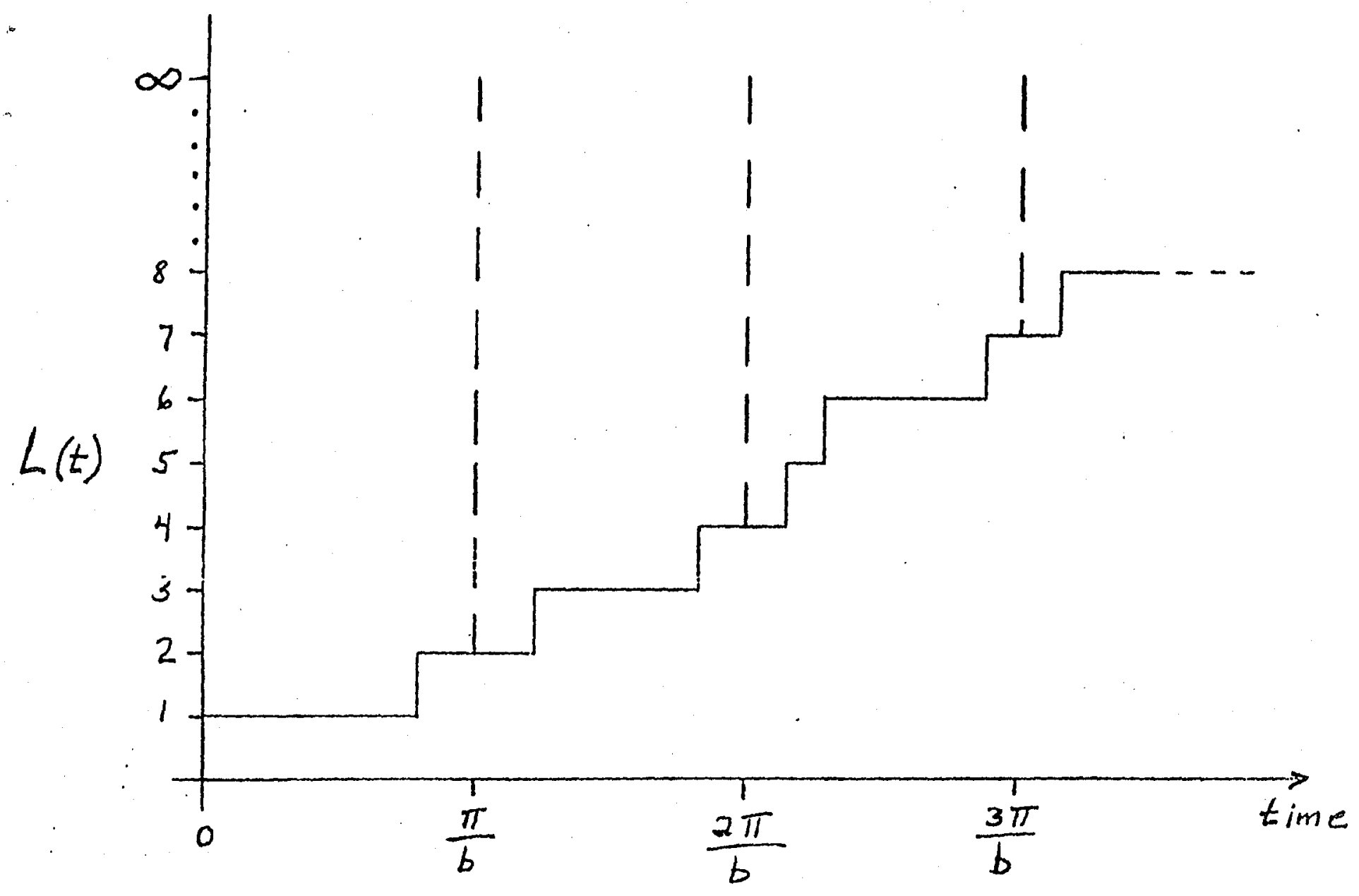


because their locations are a priori unknown. Knowledge of $\log \hat{P}\left(t_{1}\right)$ where $\hat{\mathrm{P}}\left(t_{1}\right)$ has the same structure as $\mathrm{P}(\mathrm{n} \pi / \mathrm{b})$ in the preceding illustration, can be useless for making statements about the propensity of individuals to move between particular states. 23 If many observations in time are allowed in a particular study, we could prepare sampling plans for model identification which would be relatively uninfluenced by this phenomenon. With observations at 2,3 , or 4 time points being a constraint in most studies, however, a single uninformative matrix $\hat{\mathrm{P}}\left(\mathrm{t}_{\hat{1}}\right)$ can make a conslderable difference in the available information for identifying the Q-matrix underlying a substantive process.

With general r-state matrices, the preceding discussion is complicated by the possible presence of more than one pair of complex conjugate eigenvalues. The graph of $\mathrm{L}(\mathrm{t})$ (figure 6 ) would then be altered in two ways: First, there are additional isolated time points at which $L(t)=+\infty$. These correspond to the instants at which the added complex eigenvalues have zero Imaginary parts and become repeated real roots. Second, the rise in the step function can be much steeper. This is because the wedge-shaped region (figure 4), which determines the number of branches of $\log \lambda(t)$ that can generate candidates for membership in $\varrho$, widens as a function of $r$, the order of the matrix. This phenomenon is illustrated in figures 7, 8, and 9 .

\section{Figures 7,8 , and 9 about here}

Figure 7 displays the wedge-shaped zones for general 3-state and 6-state matrices; the respective angles made with the positive real axis are determined by the inequalities (4.3). From the illustrative representation of an eigenvalue of $\log \hat{P}$ and its complex conjugate, we see that while only the principal branch lies in the wedge for $3 \times 3$ matrices, two additional branches would be candidates for membership in $\underline{\underline{Q}}$ if this same eigenvalue belonged to 
Figure 7. Runnewherg's Wedge Criterion, 111ustrated for $3 \times 3$ and Gx6 Matrleas, for $t=1$

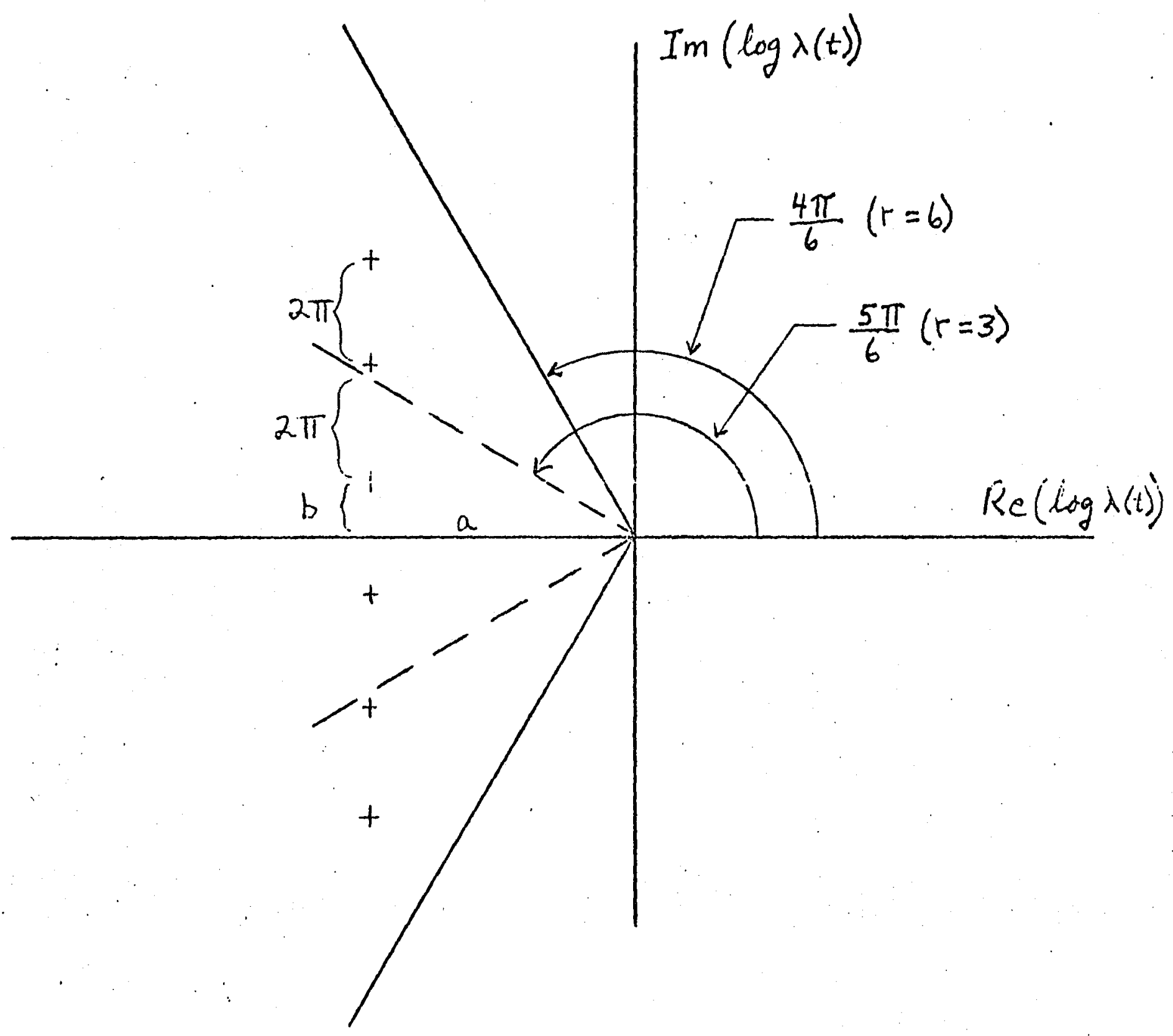


Flgure 8. Elgenvalue Reglons of $\hat{P}$ in whioh Two Verstons of log $\hat{p}$ are Candidntes for Membership in 9 , for $4 \times 4,6 \times 6,12 \times 12$, and $20 \times 20$ Matrices 1

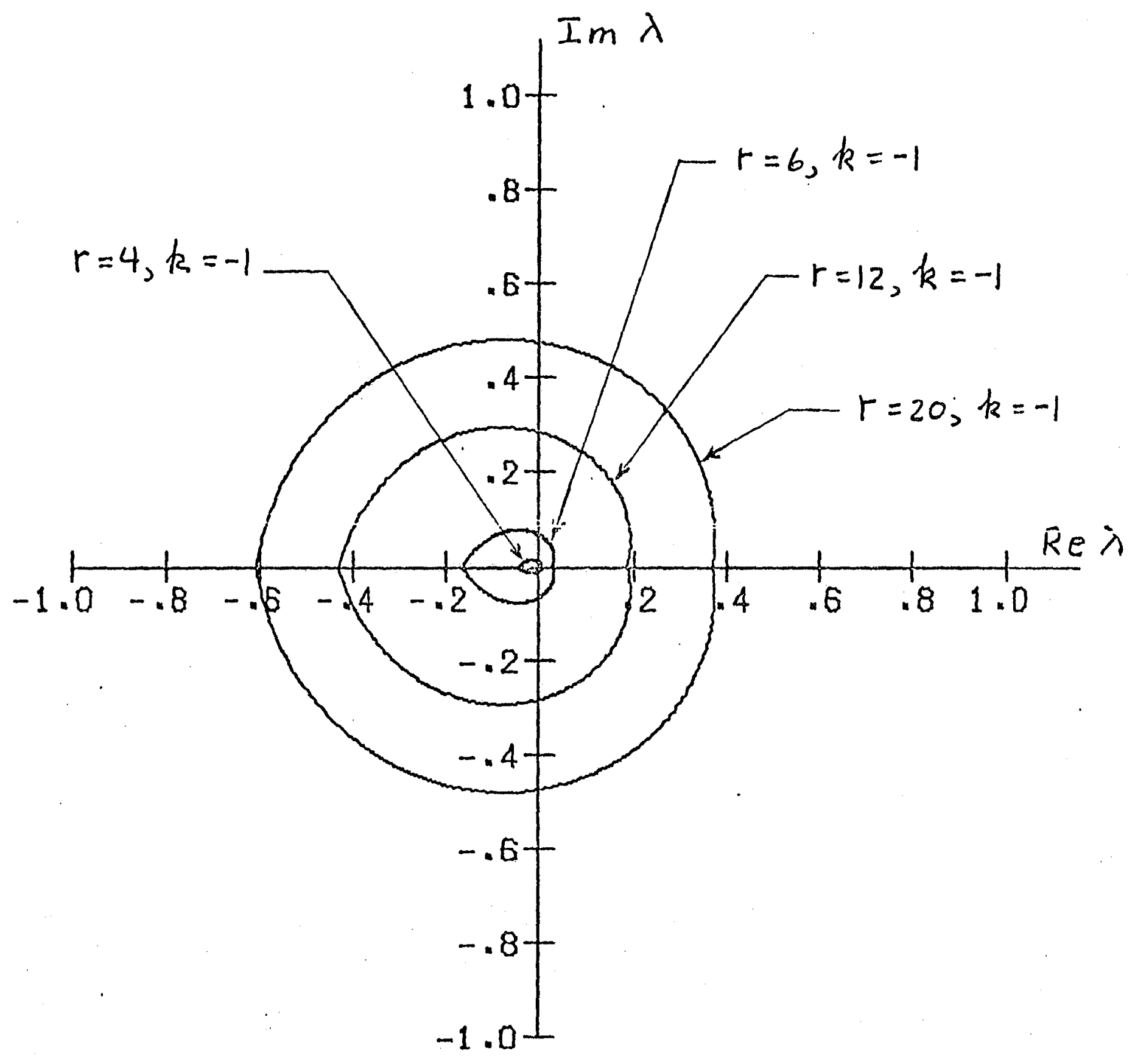

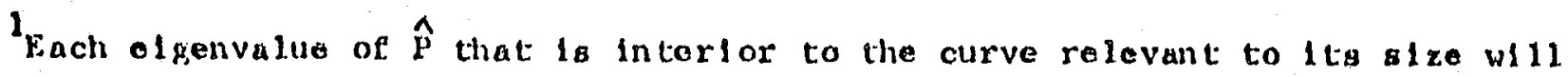
gencrate at loast two logarlthin candldates for membershlp in $Q$. 
Flgure 9. rigenvalue Reglors of $\hat{p}$ In whlch Multiple Veralong of $\log \hat{p}$ are Candidates for Memlershlp In ?, for $20 \times 20$ Matrices

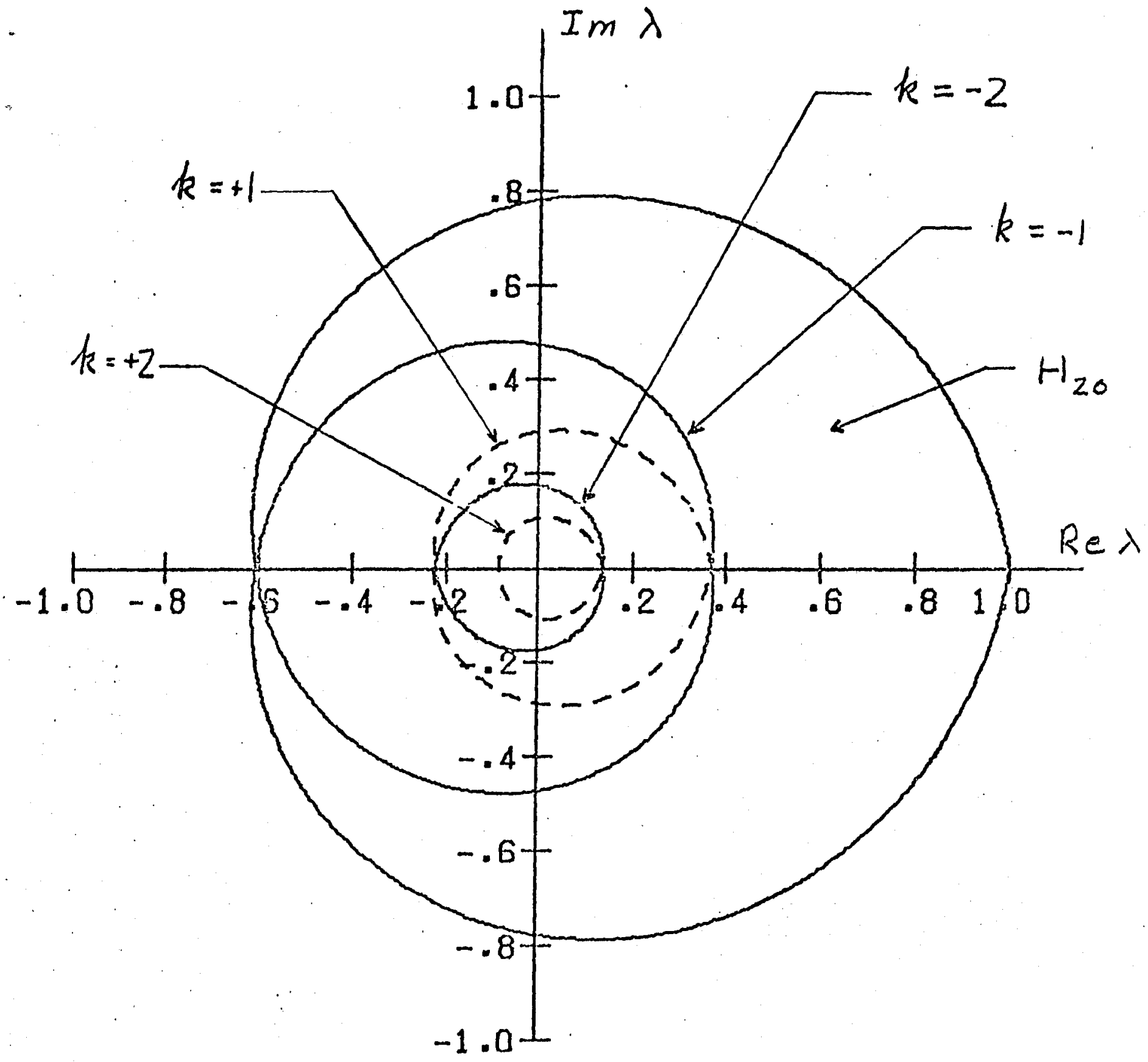

${ }^{1}$ All efgenvalues of $\hat{p}$ wast 210 in the reglan $\|_{20}$ for $\hat{p}$ to be enloddablo. It an elgenvalue la interior to a k-curve, it generates $|k|+1$ versions of $l o g \hat{p}$ Which may to in Q. 
the larger array. It is this fact, together with the presence of additional complex conjugate efgenvalues to generate candidates for membership in $\underline{Q}$, which prompted our remark in section 3.3 to the effect that the number of branches which must be checked for embeddability increases directly with the order of $\cdot \hat{P}$. In the context of the present discussion, we emphasize that the computations are more likely to produce multiple versions of $\log \hat{\mathrm{P}} \in \underline{Q}$ in large-order arrays.

Figure 8 presents the same information as figure 7, but from a different perspective. The preceding plot depicted the constraints on the brancheo of the elgenvalues of $\log \hat{\mathrm{p}}$, as they relate to eligibility for membership in $\underline{\underline{Q}}$. In figure 8 we display the conditions on the eigenvalues of $\hat{\underline{p}}$, in the case of $4 \times 4,6 \times 6,12 \times 12$, and $20 \times 20$ matrices, for 1 t to generate at

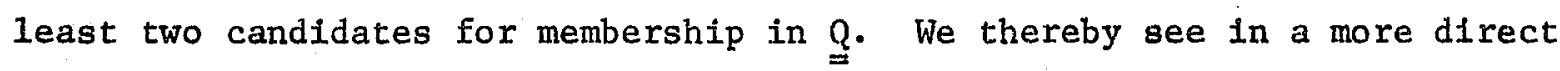
fashion how the constraints are relaxed as the matrix size is increased. 24 Finally, in figure 9 we show the restrictions for different numbers of logarithms to be eligible for membership in $Q$, in the particular instance of a $20 \times 20$ array. The outer, heart-shaped region, labeled $\mathrm{H}_{20}$, is a graph of Runnenberg's necessary conditions: 811 eigenvalues of $\hat{P}$ must 11e in this zone for the matrix to be embeddable. The interior curves delineate the regions in which an eigenvalue of $\hat{\mathrm{P}}$ will generate multiple branches of $\log \hat{\mathrm{P}}$ that can be in $\underline{\underline{Q}}$; for instance if some elgenvalue $\lambda_{j}$ lies interfor to the curve labeled " $k=-1$," then each of the two branches of its logarithm,

$$
\log \lambda_{j}=a+b i \text { and } \log \lambda_{j}=a+i(b-2 \pi)
$$

will generate versions of $\log \hat{\mathrm{P}}$ which must be examined for membership in $\underline{Q}$. The most severe form of non-uniqueness of $\log \hat{P}\left(t_{1}\right)$ occurs for Markov chains $P(t)=e^{Q t}$ having real eigenvalues which remain repeated for all 
$t>0$ rather than separating into complex conjugates, as was the case in the preceding discussion. The transition mechanisms assoclated with such chains are by no means pathological from a substantive point of view, and the prototype of this pheriomenon is illustrated in the following example. Example 13:

Consider the matrix

$$
P(t)=\frac{1}{3}\left(\begin{array}{ccc}
1+2 e^{-3 t / 2} & 1-e^{-3 t / 2} & 1-e^{-3 t / 2} \\
1-e^{-3 t / 2} & 1+2 e^{-3 t / 2} & 1-e^{-3 t / 2} \\
1-e^{-3 t / 2} & 1-e^{-3 t / 2} & 1+2 e^{-3 t / 2}
\end{array}\right)
$$

where $t>0 . P(t)$ has eigenvalues $1, e^{-3 t / 2}, e^{-3 t / 2}$ (which are repeated irrespective of the choice of $t$ ), and non-distinct elementary divisors $(\lambda-1)$, $\left(\lambda-e^{-3 t / 2}\right),\left(\lambda-e^{-3 t / 2}\right)$. Note that this is the matrix of example 10 with $x=e^{-3 t / 2}$.

From the discussion of repeated elgenvalues with non-distinct elementary divisors (section 3.2 II) we know that all branches of $\frac{1}{t} \log P(t)$ may be computed via

$$
\frac{1}{t} \log P(t)=\frac{1}{t} H B \log J(t) B^{-1} H^{-1}
$$

where $H$ is any similarity transformation that reduces $P(t)$ to diagonal form (e.g., equation 3.19), B is a matrix with complex entries (3.21) which commutes with $J(t)$, and $\log J(t)$ has the form

$$
\log J(t)=\left(\begin{array}{ccc}
0 & 0 & 0 \\
0 & \frac{-3 t}{2}+2 \pi k i & 0 \\
0 & 0 & \frac{-3 t}{2}-2 \pi k i
\end{array}\right)
$$

in which $k=0, \pm 1, \pm 2, \ldots$ specifies branches of the logarithm. 
We now describe how a continuum arises in this eigenvalue condition. The first time that the complex eigenvalues in (4.6) satisfy Runnenberg's condition (4.3) with $k \neq 0$ occurs at $t^{*}=4 \pi / \sqrt{3}$. Before this time only the branch $k=0$ of $\log J(t)$ will be in the wedge-shaped zone (figure 4). It can be checked that when $k=0, B \log J(t)=\log J(t) B$, and therefore equation (4.5) reduces to $\frac{1}{t} H \log J(t) H^{-1}$ for every matrix B. This means that at most one version of $\frac{1}{t} \log P(t)$ can be in $\underline{Q}$. Indeed,

$$
Q=\frac{1}{t} \log P(t)=\left(\begin{array}{ccc}
-1 & \frac{1}{2} & \frac{1}{2} \\
\frac{1}{2} & -1 & \frac{1}{2} \\
\frac{1}{2} & \frac{1}{2} & -1
\end{array}\right) \quad \text { for } 0<t<\frac{4 \pi}{\sqrt{3}}
$$

When $t>t^{*}=\frac{4 \pi}{\sqrt{3}}$ a second branch of $\log J(t)$ in (4.6) enters the wedgeshaped zone (see figure 5). In this circumstance, it is no longer the case that $B \log J(t)=\log J(t) \mathrm{Y}$, and a continuum of versions of $\frac{1}{t} \log P(t)$ wil1 be generated, each version corresponding to a choice of $\left\{c_{i j}\right\}$ in $B$ (equation 3.21). A bit of computation will show that if $\left\{c_{i j}\right\}$ are restricted according to

(i) $c_{11} c_{12}-c_{21} c_{22}=0$

(ii) $u$ is real, $u=\frac{c_{11} c_{22}+c_{12} c_{21}+2 c_{21} c_{22}}{c_{11} c_{22}-c_{12} c_{21}}$

$$
v \text { is real, } v=\frac{c_{11} c_{22}+c_{12} c_{21}+2 c_{21} c_{22}}{c_{11} c_{22}-c_{12} c_{21}}
$$

(iii) $|\mathrm{ku}|<\sqrt{3} \mathrm{t} / 4 \pi$ and $|\mathrm{kv}|<\sqrt{3} \mathrm{t} / 4 \pi$

where $k$ is an integer (the branch number), then all choices of $\left\{c_{1 j}\right\}$ will yield matrices $Q \in \underline{Q}$, and they are summarized by 
$Q=\frac{1}{t} \log P(t)=\left(\begin{array}{ccc}-1 & \frac{1}{2}+\frac{2 k \pi}{\sqrt{3}} \frac{u}{t} & \frac{1}{2}-\frac{2 k \pi}{\sqrt{3}} \frac{u}{t} \\ \frac{1}{2}-\frac{2 k \pi}{\sqrt{3}} \frac{v}{t} & -1-\frac{k \pi}{\sqrt{3}} \frac{(u-v)}{t} & \frac{1}{2}+\frac{k \pi}{\sqrt{3}} \frac{(u+v)}{t} \\ \frac{1}{2}+\frac{2 k \pi}{\sqrt{3}} \frac{v}{t} & \frac{1}{2}-\frac{k \pi}{\sqrt{3}} \frac{(u+v)}{t} & -1+\frac{k \pi}{\sqrt{3}} \frac{(u-v)}{t}\end{array}\right)$ for $t>\frac{4 \pi}{\sqrt{3}}$

The graph of $L(t)$ vs. $t$ for this Markov chain is shown in flgure 10 . From the point of view of model identification, the second observation $t_{1}$ must be taken before $t^{*}=4 \pi / \sqrt{3}$. After this time, a repeated observation will yield the matrix (4.8) which is completely uninformative about the propensity to move between different states. The fundamental difficulty illustrated by this example is that empirically determined matrices with non-distinct elementary divisors in which this property is retained through time may be associated with a continuum of intensity matrices for all times $\mathrm{t}_{1}$ greater than some threshold $t^{*}$. To distinguish this "essential" continuum case from the chance occurrence of an "isolated" continuum (viz. the points $\frac{\pi}{b}, \frac{2 \pi}{b}, \ldots$, $\frac{\mathrm{n} \pi}{\mathrm{b}}, \ldots$ in section 4.2 ) a researcher should check whether the eigenvalues of $\hat{\mathrm{P}}\left(t_{1}+\Delta t\right)$, some $\Delta t>0$, are repeated with non-distinct elementary divisors when his initial matrix $\hat{P}\left(t_{1}\right)$ has these properties.

\section{Figure 10 about here}

\subsection{Summary of Correspondence between Eigenva1ue Gharacteristics and} Number of Matrices $Q \varepsilon Q$

The number of versions of $\log \hat{\mathrm{P}}$ that can possibly be in $Q$, as this relates to the eigenvalue characteristics of $\hat{\mathrm{P}}$, is summarized in table 1 . The left tab of the table refers to a single eigenvalue of $\hat{P}$ or to $a$ set 


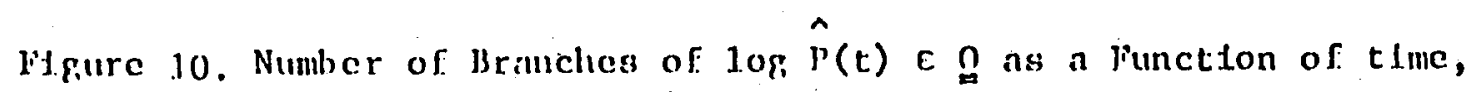
for $\hat{\mathrm{P}}(\mathrm{t})$ in Fxample 13

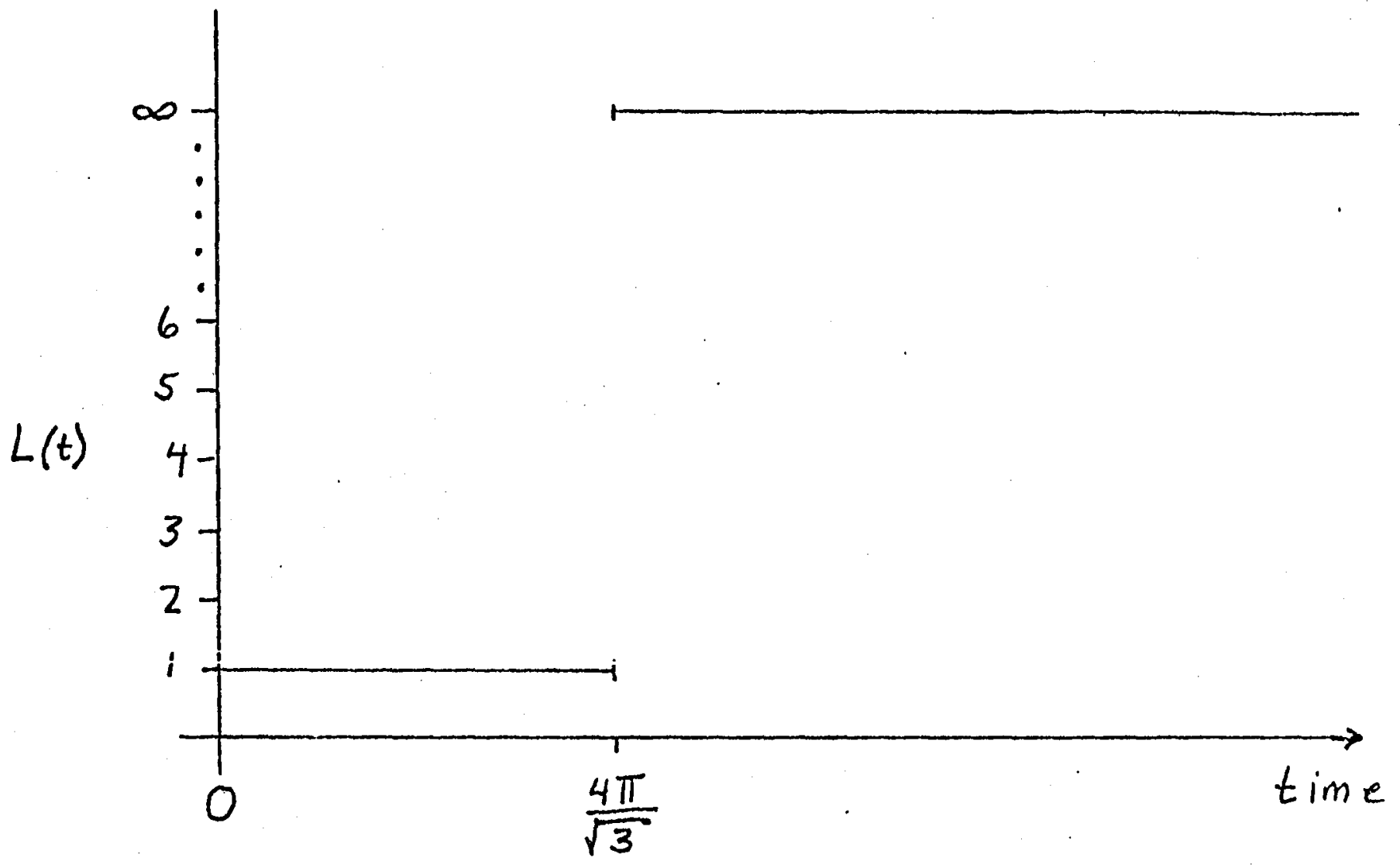


of efgenvalues sharing a common property (e.g., complex confugates). The evaluation in column 3 assumes that embeddability is met, $1 . e$. , at least one version of $\log \hat{P}$ is in $\underline{Q}$. In making this evaluation, it is also presumed that the remaining eigenvalues of $\hat{P}$ do not satisfy a condition which is compatible with a greater number of candidates for membership in 9 ; for instance, all eigenvalues must belong to categorles (1) and (2) in order to conclude, on the basis of an examination of eigenvalues alone, that at most one version of $\log \hat{P}$ is in $\underline{Q}$.

A second point to be noted in connection with the table is that the efgenvalue conditions which rule out embeddability do so by not being compatible with a real-valued version of $\log \hat{P}$. For example, if $\hat{P}$ has a unique negative eigenvalue, $\lambda=-a:(a>0)$, its logarithm will be $\log a+1 k \pi, k=0$, $\pm 1, \pm 2, \ldots$, which always has a non-zero imaginary part. The corresponding efgenvector $h$ in the similarity transformation $\hat{\mathrm{P}}=\mathrm{HJH}^{-1}$ will be realvalued (since $\lambda=-\mathrm{a}$ is distinct and real), and $\log \hat{\mathrm{P}}=\mathrm{H} \log \mathrm{JH}^{-1}$ w1Il have the identical eigenvector corresponding to its complex elgenvalue. There is no way in which $\log \hat{\mathrm{P}}$ can be real-valued in this circumstance. What alters the situation in the case of repeated negative eigenvalues with even multiplicity is that when the elementary divisors are not distinct, the elgenvectors corresponding to the repeated eigenvalues w11l be complex conjugates, and real versions of $\log \hat{\mathrm{P}}$ can result. In particular, this will occur when different branches of the logarithm of -a are present simultaneously in $\log \mathrm{D}$.

Finally, we emphasize that the elgenvalue configurations most commonly found in empirically determined matrices involve combinations of distinct posltive and distinct complex conjugates, i.e., categories (1) and (7). 
Table 1 about here

\section{TESTING 'STRATEGIES}

\subsection{Identification of Structural Parameters}

We assume first that the process under observation is time-stationary, that the data are free of measurement and classiflcation error, and that the entire population has been surveyed so sampling variability is not a concern. These assumptions have also been made, though without being noted explicitly, in the preceding sections. In this environment, the identification problem arises when observations are taken at only two time polnts $\left(t=0, t=t_{1}\right)$, and the matrix $\hat{P}\left(t_{1}\right)$ constructed from these observations can be represented in the form $\hat{P}\left(t_{1}\right)=e^{Q t_{1}}$ for multiple arrays $Q \varepsilon \varrho$. A researcher then has the following options:

(i) He may bring to bear other information about the substantive process. For instance, if $\hat{P}\left(t_{1}\right)$ were the matrix in example 12, a researcher might have reason to believe that $q_{12}>q_{14}$ and therefore $Q_{2}$, not $Q_{1}$, governs the evolution of the process. Clearly, such a choice can be made only when there is a finite list of intensity matrices, and not when a continuum is present.

(i1) If an opportunity exists to collect data at a third time point, it should be selected so as not to be an integer multiple of the initial interval $\left(0, t_{1}\right)$. The reason is because at multiples of an observation interval the same list of Q-matrices can reappear; this was the case, for instance, with the times $\pi / b, 2 \pi / b$, etc. in figure 6 . If, however, the third observation is taken at $t_{2} \neq k t_{1}, k$ an integer, ${ }^{25}$ then even in the presence of multiple branches of $\log \hat{P}\left(t_{1}, t_{2}\right) \varepsilon \underline{Q}$, only one version of the logarithm, $Q_{0}$, will have the property 
TABLE 1

Eigenvalues of $\hat{P}$ and the Number of Matrices $Q \varepsilon \underline{\underline{Q}}$

EIgenvalue Characteristics

1. positive, distinct

2. positive, repeated, distinct elementary divisors

3. positive, repeated, nondistinct elementary divisors

4. negative, distinct

5. negative, repeated, odd multip1icity

6. negative, repeated, even multiplicity

7. complex, distinct, member of a conjugate palr

8. complex conjugate, repeated

9. mixture of the above types
Embeddable?

possibly

possibly

possibly

never

never

possibly

possibly

possibly

possibly
How Many Q's?

one or continuum

continuum

one or multiple

one, multiple or continuum

the most extreme form of nonunlqueness present in any component of the mixture 


$$
Q_{0}=\frac{1}{t_{1}} \log \hat{P}\left(0, t_{1}\right)=\frac{1}{t_{2}-t_{1}} \hat{P}\left(t_{1}, t_{2}\right)
$$

This correspondence will identify the unique $Q$ that can be associated with the empirical process.

There is an additional virtue in collecting data at three or more time points. The embeddability problem concerns only the question of compat1b1lity of a single stochastic matrix $\hat{P}\left(t_{1}\right)$--i.e., observations at two time points-with a continuous-time Markov process. We have seen that on the basis of this information alone it is frequently possible to rule out a Markov structure. However, when data are available from more than two time points, a direct test can also be made of the fundamental dynamic assumption of a first-order Markov process, namely that the future state of the system depends only on current state, not on 1 ts history. These additional necessary conditions are specified by tests of the sort

$$
\hat{P}\left(t_{1}, t_{k}\right)=\hat{P}\left(t_{i}, t_{j}\right) \hat{P}\left(t_{j}, t_{k}\right), \quad 0<t_{1}<t_{j}<t_{k}
$$

The availability of data at three time points provides the most rudimentary opportunity to check this assumption. Formal statistical tests of the validity of the Markov property are described in Anderson and Goodman (1957) and Billingsley (1961).

Study design considerations. The potential for non-uniqueness can be minimized at the study design stage. If the use of Markov models is contemplated, the survey times should be chosen close together in time, while still permitting a representative amount of movement to take place. When the number of states is small (say $r \leq 5$ ) it should be possibie to select $t_{1}$ so that $\min _{i}\left\{\hat{p}_{1 i}\left(t_{1}\right)\right\}>\frac{1}{2}$. If $\hat{P}\left(t_{1}\right)$ is embeddable, this condition on the 
dlagonal elements ensures that $\log \hat{\mathrm{P}}\left(\mathrm{t}_{1}\right) \varepsilon \underline{\underline{Q}}$ will be unique (see section 4.1). When the number of system states is large, it may not be possible to satisfy this condition and still retain an adequate amount of population movement to estimate $\log \hat{\mathrm{P}}\left(t_{1}\right)$ accurately. Even in this circumstance, however, $t_{1}$ should be selected reasonably close in time to the inftial observation, since the degree of non-uniqueness of $Q \varepsilon \varrho$ is a monotone increasing function of time (figure 6 ), except for isolated instants such as $\{k \pi / b\}$.

In most data gathering situations one has neither a priorf information concerning the rate of movement (to assist in selecting the second observation), nor an opportunity to schedule the second wave of a survey according to these considerations. A more pragmatic suggestion would be to collect detailed retrospective information about the process. Ideally, this should consist of "sample path" data; that 1s, complete information about a respondent's duration in each system state over the time interval of interest. Where such data are deemed too costly to collect, a respondent should be queried regarding his system state at several pre-chosen time points in the past (e.g., one year ago, two years ago, etc.). Having gathered such information the estimation procedures and model tests that require more than two observations in time may be utilized.

\subsection{Sampling Error and Data Noise}

The data avallable to researchers are commonly contaminated by errors of various sorts. While we may wish to make statements about a populationlevel process, information is usually collected for a population sample. Similarly, errors of measurement can result in the misclassification of Individuals with respect to system state.

Ordinarily, these are not very serious problems. In many sampling situations the inference made about a population parameter, using standard 
statistical procedures, tends to be incorrect to a degree that varies continuously with the magnitude of the measurement error. By using distributional statistics, one can put confidence bounds around an estimate, and describe the interval in which the population-level parameter lies. However, measurement error and sampling varlability carry greater consequence when we seek to Identify the non-directly observable structural mechanlsms (Q-matrices) that underly Markov processes. In particular, when an empirically determined matrix $\hat{\mathrm{P}}\left(\mathrm{t}_{1}\right)$ is in the vicinity of a second stochastic matrix $\widetilde{\mathrm{P}}$ which can be expressed in the form $\tilde{\mathrm{P}}=\mathrm{e}^{\mathrm{Q}}$ for multiple versions of $\log \tilde{\mathrm{P}}_{\varepsilon} g$, then a small error in the estimate of $\hat{P}\left(t_{1}\right)$ can result in the recovery of a matrix $Q \varepsilon \underline{Q}$ which, while unique, is the wrong intensity matrix for the substantive process. Examp1e 13:

Suppose you observe

$$
\hat{\mathrm{p}}_{1}\left(t_{1}\right)=\left(\begin{array}{llll}
.232 & .249 & .266 & .253 \\
.254 & .236 & .242 & .268 \\
.270 & .258 & .228 & .244 \\
.245 & .274 & .250 & .231
\end{array}\right)
$$

This matrix can be written in the form $e^{Q t_{1}}$ for a unique version of $\log \hat{\mathrm{P}}\left(t_{1}\right) \in \underline{Q}$,

$$
Q_{1}=\left(\begin{array}{rrrr}
-3.216 & .129 & .064 & 3.023 \\
3.007 & -3.174 & .138 & .029 \\
.034 & 3.104 & -3.260 & .122 \\
.132 & .032 & 3.023 & -3.186
\end{array}\right)
$$

If one believes that $\hat{\mathrm{P}}_{1}\left(t_{1}\right)$ is error free, it would be reasonable to conclude that $Q_{1}$ describes the evolution of the dynamic process. However, in a fallible environment, a second survey of the same population would produce a slightly different observed matrix. Consider, 


$$
\hat{\mathrm{P}}_{2}\left(\mathrm{t}_{1}\right)=\left(\begin{array}{llll}
.231 & .255 & .266 & .248 \\
.250 & .234 & .247 & .269 \\
.271 & .252 & .227 & .250 \\
.251 & .275 & .245 & .229
\end{array}\right)
$$

No element of this matrix differs from its counterpart in $\hat{\mathrm{P}}_{1}\left(\mathrm{t}_{1}\right)$ by an amount in excess of .006 in magnitude, so it is not unreasonable to suggest that the two matrices represent different samples from a single parent population. However, while $i t$ is the case that $\hat{\mathrm{P}}_{2}\left(t_{1}\right)$ is also compatible with a continuous-time Markov process for a unique $Q \varepsilon g$, this intensity matrix is given by

$$
Q_{2}=\left(\begin{array}{rrrr}
-3.164 & 3.148 & .005 & .011 \\
.031 & -3.170 & 3.049 & .090 \\
.015 & .022 & -3.167 & 3.130 \\
3.130 & .048 & .026 & -3.204
\end{array}\right)
$$

Matrices $Q_{1}$ and $Q_{2}$ represent very different structural mechanisms, and would lead to contrary conclusions about the nature of the substantive process: What has happened is that whtle $\hat{P}_{1}\left(t_{1}\right)$ and $\hat{P}_{2}\left(t_{1}\right)$ are each compatible with the representation $e^{Q t_{1}}$ and have unique logarithms in $g$, the two empirically determined P-matrices lie in the viclnity of a third, $\widetilde{P}$, which in turn can be represented as a Markov process for multiple matrices $Q \varepsilon \varrho$. Indeed,

$$
\begin{aligned}
& \tilde{P}=\hat{P}_{1}\left(t_{1}+.05\right)=e^{\left(t_{1}+.05\right) Q_{1}} \\
& \tilde{P}=\hat{P}_{2}\left(t_{1}+.05\right)=e^{\left(t_{1}+.05\right) Q_{2}}
\end{aligned}
$$

and this common P-array is the same one presented in example 12 to 111 ustrate the phenomenon of multiple intensity matrices. 26 
Specific error structures. In the context of sampling variability or measurement error, then, a researcher cannot assume that because $\hat{P}\left(t_{1}\right)=e^{Q t_{1}}$ for a unique $Q \in Q Q$, this intensity matrix describes the evolution of the substantive process. He must either remove the error from the observed matrix and use the "purged" array for estimating structural parameters, or examine the intensity matrices of other $P^{\prime} s$ that are within "error distance" of his empirically determined matrix.

Misclassification error can be formally incorporated in a description of observed transition matrices by introducing the representation

$$
\hat{P}\left(t_{i}, t_{j}\right)=\bar{P}\left(t_{i}, t_{j}\right) \circ \varepsilon\left(t_{i}, t_{j}\right) \quad 0 \leq t_{i}<t_{j}
$$

where $\hat{\mathrm{P}}\left(t_{1}, t_{j}\right)$ is an empirically determined $r \times r$ matrix of transition probabilities based on observations at times $t_{1}$ and $t_{j} ; \bar{P}\left(t_{1}, t_{j}\right)$ is a fitted $r \times r$ matrix of transition probabilities representing the error-free or purged mobility structure; $\varepsilon\left(t_{1}, t_{j}\right)$ is an $r \times r$ matrix of residuals interpreted as errors due to misclassification; and the symbol "o" denotes etther the operation addition or multiplication. Motivating the representation (5.3) is the view that matrix $\bar{P}$, rather than $\hat{P}$, should be tested for compatibility with a Markov process, and $Q$ should be estimated from the equation $\bar{P}=e^{Q}$.

Calculation of $\overline{\mathrm{P}}$ and $\varepsilon$ must be based on an assumed model of the error structure, together with independent estimates of the parameters. For example, If the states are occupational categories and there is a natural ordering among them (e.g., on the basis of a prestige scale), an individual who actually moves from state $i$ at time $t_{1}$ to state $j$ at time $t_{2}$ may have probability $c_{1}$ of being recorded in state $j-1$ at time $t_{2}$, probability $c_{2}$ of being recorded in state $j+1$ at time $t_{2}$, and probability $1-c_{1}-c_{2}$ of being recorded correctly. If this kind of measurement error is believed to operate, then it implies a 
representation of the form

$$
\hat{P}\left(t_{1}, t_{2}\right)=\bar{P}\left(t_{1}, t_{2}\right) c
$$

where

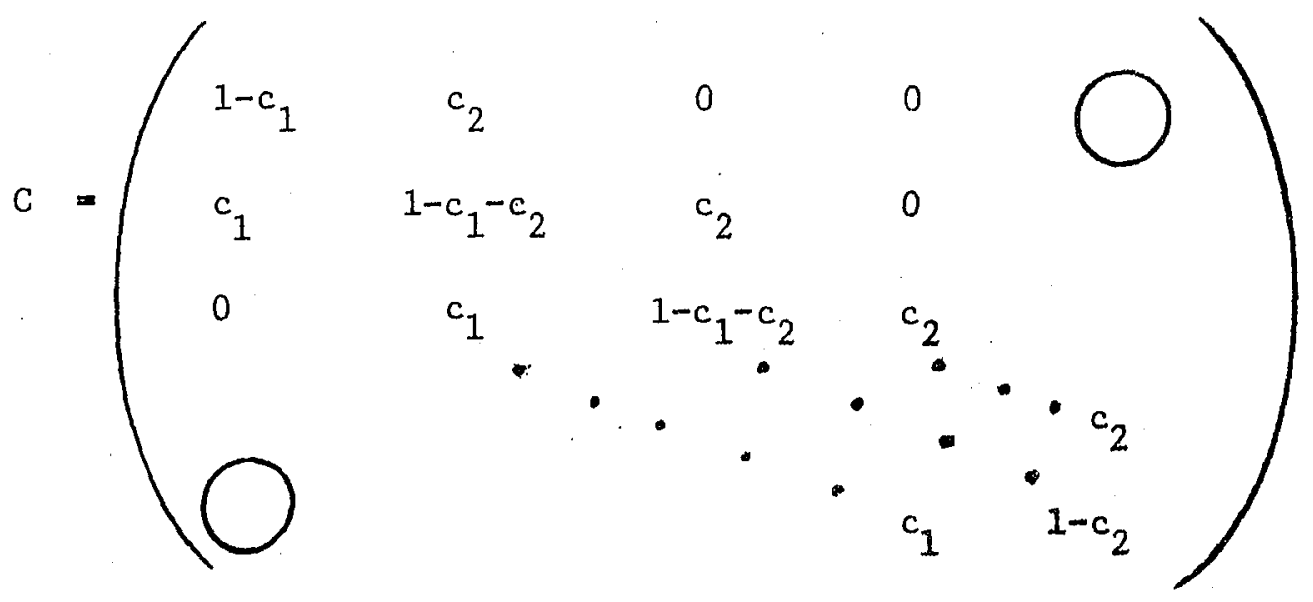

Given $c_{1}$ and $c_{2}$ based on independent misclassification estimates, we could solve the matrix equation (5.4) for $\overline{\mathrm{P}}\left(\mathrm{t}_{1}, \mathrm{t}_{2}\right)$. See Coleman (1964b) for approaches of this sort to the study of change in a fallible environment.

Random error. In genera1, a formal model of the error structure will not be available, yet we may wish to make allowance for the effect of "noise" in the data. We recommend a strategy of "exploring" a neighborhood of the observed matrix $\hat{P}\left(t_{1}\right)$, to ascertain whether nearby $\mathrm{P}$-arrays are compatible with intensity matrices that are very different from the initial Q-matrix.

A reasonable procedure for exploring a neighborhood of $\hat{P}\left(t_{1}\right)$ would be' to "rlde" its associated intensity matrlx $Q_{0}$. By this is meant computing $P(t)$ from the representation $P(t)=e^{Q_{0} t}$ using for $t$ the values $t_{1}-\Delta t, t_{1}-2 \Delta t$, $\ldots, t_{1}-h \Delta t$, and $t_{1}+\Delta t, t_{1}+2 \Delta t, \ldots, t_{1}+k \Delta t$, where the termination points $h$ and $k$ are the last times that $P\left(t_{1}-j \Delta t\right)$ and $P\left(t_{1}+j \Delta t\right)$ can be considered "within sampling or measurement error" of the observed matrix. Next, examine the eigenvalues in the sequence of matrices. 
(a) If there is a complex conjugate pair $(\lambda, \vec{\lambda})=(a \pm b i)$ whose imaginary part passes through zero, then $\hat{\mathrm{P}}\left(\mathrm{t}_{1}\right)$ is in a neighborhood of some matrix $\tilde{\mathrm{P}}$ which has repeated real eigenvalues. Associated with this array, a continuum of matrices $Q \varepsilon \varrho$ will satisfy the relation $\widetilde{P}=e^{Q}$. Strategies for exploring the structure of a continuum are discussed in Appendix 1.

(b) If a continuum does not occur within error distance, recover all matrices $Q \in \underline{\underline{Q}}$ that are compatible with the representation $P\left(t_{1}+k \Delta t\right)$ $=e^{Q_{0}\left(t_{1}+k \Delta t\right)}$, where $k$ was chosen as the forward stopping point of the sequence of P-matrices. 27 The complete solution to the problems of determining the number of candidates for membership in $\underline{Q}$, and computing all versions of $\log P \varepsilon g$, was presented in section 3 .

If It is the case that $\log \hat{P} \varepsilon \underline{Q}$ is unique under the perturbations of $\hat{\mathrm{P}}\left(t_{1}\right)$, then this intensity matrix can be viewed as the sole moblitiy structure compatible with a Markov formulation of the substantive process. Stated more transparently, additional samples from the same population can be expected to produce similar Q-matrices. In contrast, if multiple mobility mechanisms $Q \varepsilon Q$ are found for matrices $P$ within error distance of the observed array $\hat{\mathrm{P}}\left(\mathrm{t}_{1}\right)$, then one of the procedures described in section 5.1 for selecting among alternative intensity matrices must be utilized.

In an environment containing error, the advantages of collecting data at three or more points in time are especially apparent. We noted earlier (section 5.1) that three time points is the minimum number for a direct test of the dynamic assumption underlying a first-order Markov process, 1.e., checking that

$$
\hat{P}\left(0, t_{2}\right)=\hat{P}\left(0, t_{1}\right) \hat{P}\left(t_{1}, t_{2}\right), \quad 0<t_{1}<t_{2}
$$


In practice, this entails evaluating whether $\left\|\hat{\mathrm{P}}\left(0, t_{2}\right)-\hat{\mathrm{P}}\left(0, t_{1}\right) \hat{\mathrm{P}}\left(\mathrm{t}_{1}, t_{2}\right)\right\|<\varepsilon$, for $\varepsilon>0$, and some suitably chosen norm [e.g., $\|A\|=\sqrt{\left.\sum_{1, j} a_{1 j}^{2}\right]}$. When (5.5) is satisfied, and it is also the case that

$$
\frac{1}{t_{1}} \log \hat{\mathrm{P}}\left(0, t_{1}\right) \approx \frac{1}{t_{2}-t_{1}} \log \hat{\mathrm{P}}\left(t, t_{2}\right) \approx \frac{1}{t_{2}} \log \hat{\mathrm{P}}\left(0, t_{2}\right)
$$

we would define $Q$, the common intensity matrix for the process, as an average of these three estimates. In the presence of sampling or measurement error, then, data at three or more time points permits a test of the fundamental Markov assumption and also facilitates an accurate calculation of $Q$, through the pooling of several estimates.

In an instance where (5.5) is satisfled, but equation (5.6) is not, the process will still be Markovian, though it no longer is time-stationary. This leads to the problem of testing observed matrices for compatibility with a time-homogeneous Markov model (the null hypothesis) against special non-timehomogeneous alternatives. We hope to discuss this important issue in a future publication.

As a final comment on analytic strategy in the context of data noise, we emphasize that while the occurrence of multiple matrices $Q \varepsilon Q$ may not be very common in an error-free environment, it characterizes the normal work sttuation when data are fallible. This is because we advise a researcher to examine a neighborhood of an observed $\hat{\mathrm{P}}\left(t_{1}\right)$ for the presence of additional Intensity matrices, and to consider each recovered $Q \varepsilon Q$ as possibly governing the evolution of the empirical process. Due to data nolse, then, we suggest creating a multiple $Q \varepsilon \underline{Q}$ situation when an observed transition matrix has associated with it a unique logarithm in $Q$. For this reason, collection of data at three or more time points should be a routine requirement when the use of Markov models is contemplated. 


\section{CONCLUSIONS}

The point of departure for this study was the gross misunderstanding among researchers concerning which stochastic matrices are compatible with a continuous-time Markov process having stationary transition probabilities. We noted that the power series representation of the logarithm of a matrix [equation (2.5)]--the principal formula used in estimating the structural parameters that govern the evolution of a Markov process--permits an Intensity matrix to be recovered only for a subset of this class of stochastic models. By resorting, instead, to the spectral decomposition representation, we were able to estimate intensity matrices for Markov models in instances where (2.5) does not converge; that is, in cases of transition arrays which Coleman and others have considered not to be compatible with this mathematical structure. In the course of the investigation, we also raised new issues which a researcher must consider; these include, principally, the possibility that multiple intensity matrices may be compatible with an empirically determined transition array, and the fact that, as a result of data "notse," recovery of a unique $Q \varepsilon \underline{\underline{Q}}$ does not preclude the possibility that the observed process is governed by an entirely different intensity matrix.

In subsequent papers we intend to address two additional issues which a researcher desiring to use Markov models in a flexible and creative manner must entertain: (a) how should a priori restrictions be placed on the elements of a Q-matrix, and (b) how can a researcher discriminate among the alternative mathematical models which, on substantive grounds, provide reasonable descriptions of his data. The first topic was mentioned, in passing, in section 3.3 , when we sought to adjust a non-embeddable $\log \hat{\mathrm{P}}$ to a neighboring $Q \in \underline{Q}$. More generally, we may wish to estimate the parameters 
of a sociological theory which specifies that certain instantaneous transitions are prohibited (see Coleman 1964a, chaps. 4 and 5 for examples). The second topic refers to testing data for compatibility with a subset of Markov models (such as birth and death processes) versais general finitestate Markov processes, and to comparing the fit of Markov models with that of other mathematical structures, such as mixtures of Markov processes or semi-Markov processes.

As a final point, we emphasize that the problems addressed in this paper cannot be avoided by employing a discrete-time Markov framework in place of a continuous-time formulation. In the discrete-time model the counterpart to the task of estimating $Q \varepsilon Q$ entalls recovering the one-step transition matrix for an empirical process, 1.e., taking the appropriate roots of the observed matrix $\hat{P}$. Like a logarithm, a root is a multiple-valued function, so the problem of non-uniqueness which we have discussed here also arlses in that formulation. Conceptually, the discrete-time model embodies a further difficulty: Because most social processes evolve continuously, there usually isn't a compeliing reason for preferring ane specification of the untt time interval to another. (For instance, in studying Intra-generational occupational moblity, should the unit time interval be five years or three years or six. months?) Yet, this is a question of great consequence because an emplrically determined matrix (estimated, let us say for this 1llustration, from observatIons ten years apart) may be consistent with a discrete-time Markov structure for some cholces of the unit time Interval but not for other choices (see Singer and Spllerman 1974, pp. 360-363 for an example). Where no substantive meaning can be attached to a particular interval length, this does not imply that the unit time interval can be specifled at the conventence 
of the researcher, or that tests of the sort described here can be Ignored. Rather, it suggests that the appropriate mathematical structure is a continuous-time formulation, the procedures for which have been discussed in this paper. 
APPENDIX 1. Exploring a Continuum

In the case where $\hat{\mathrm{P}}$ has repeated eigenvalues and non-distinct elementary divisors, the value of $\log \hat{\mathrm{P}}$ depends on the cholce of similarlty transformation that is used to reduce $\hat{\mathrm{P}}$ to Jordan form. A computer based strategy to test a representative collection of branches of $\log \hat{P}$ for membership in $\underline{Q}$ is the most direct approach we can currently recommend for deciding on compat1bility of $\hat{\mathrm{P}}$ with a continuous-time Markov model. If a branch of $\log \hat{\mathrm{P}}$ which belongs to $\varrho$ is discovered during the computer tests, then 1 t can be shown that there is in fact a continuum of branches which are in $\mathrm{g}$. The testing strategy outlined below, and illustrated in a simple example, is also designed to give some indication of the extent of the continuum of branches which are in $\underline{Q}$.

Step 1. Compute one similarity transformation $H$ which reduces $\hat{P}$ to Jordan form. The method of computation is entirely at the discretion of the researcher (see Gantmacher [1960, chap. 6] for suggestions).

Step 2. Take a random sample of points in an 8-dimensional square region with center at the origin. ${ }^{28}$ For each sample of 8 numbers, use them as the real and imaginary parts of the parameters in the matrices $B$ which commute with $\mathrm{J}=\mathrm{H}^{-1} \hat{\mathrm{P}} \mathrm{H}$. Then evaluate

$$
\log \hat{\mathrm{P}}=\mathrm{HB} \log \mathrm{J} \mathrm{B}^{-1} \mathrm{H}^{-1}
$$

where

$$
B=\left(\begin{array}{ccc}
1 & 0 & 0 \\
0 & c_{11} & c_{12} \\
0 & c_{21} & c_{22}
\end{array}\right), \quad \log \mathrm{J}=\left(\begin{array}{ccc}
0 & 0 & 0 \\
0 & \log \lambda_{2} & 0 \\
0 & 0 & \overline{\log \lambda_{2}}
\end{array}\right),
$$

the $\left\{c_{i j}\right\}$ are given by 


$$
\begin{array}{ll}
c_{11}=x_{11}+1 y_{11} & c_{12}=x_{12}+i y_{12} \\
c_{21}=x_{21}+i y_{21} & c_{22}=x_{22}+i y_{22}
\end{array}
$$

$\left\{x_{i j}\right\},\left\{y_{i j}\right\}$ are the 8 numbers associated with each sample point, and $\overline{\log \lambda_{2}}$ denotes the complex conjugate of $\log \lambda_{2}$. Note whether this branch of $\log \hat{\mathrm{p}}$ is in $\underline{Q}$. Several hundred such evaluations may be necessary in order to identify those matrice $B$, if any, which yleld versions of $\log \hat{P} \in Q$.

The preceding computations do not increase in complexity for $r \times r$ matrices having a single pair of repeated real roots, which is the situation most likely to arise. In this general case, $B$ will have the form

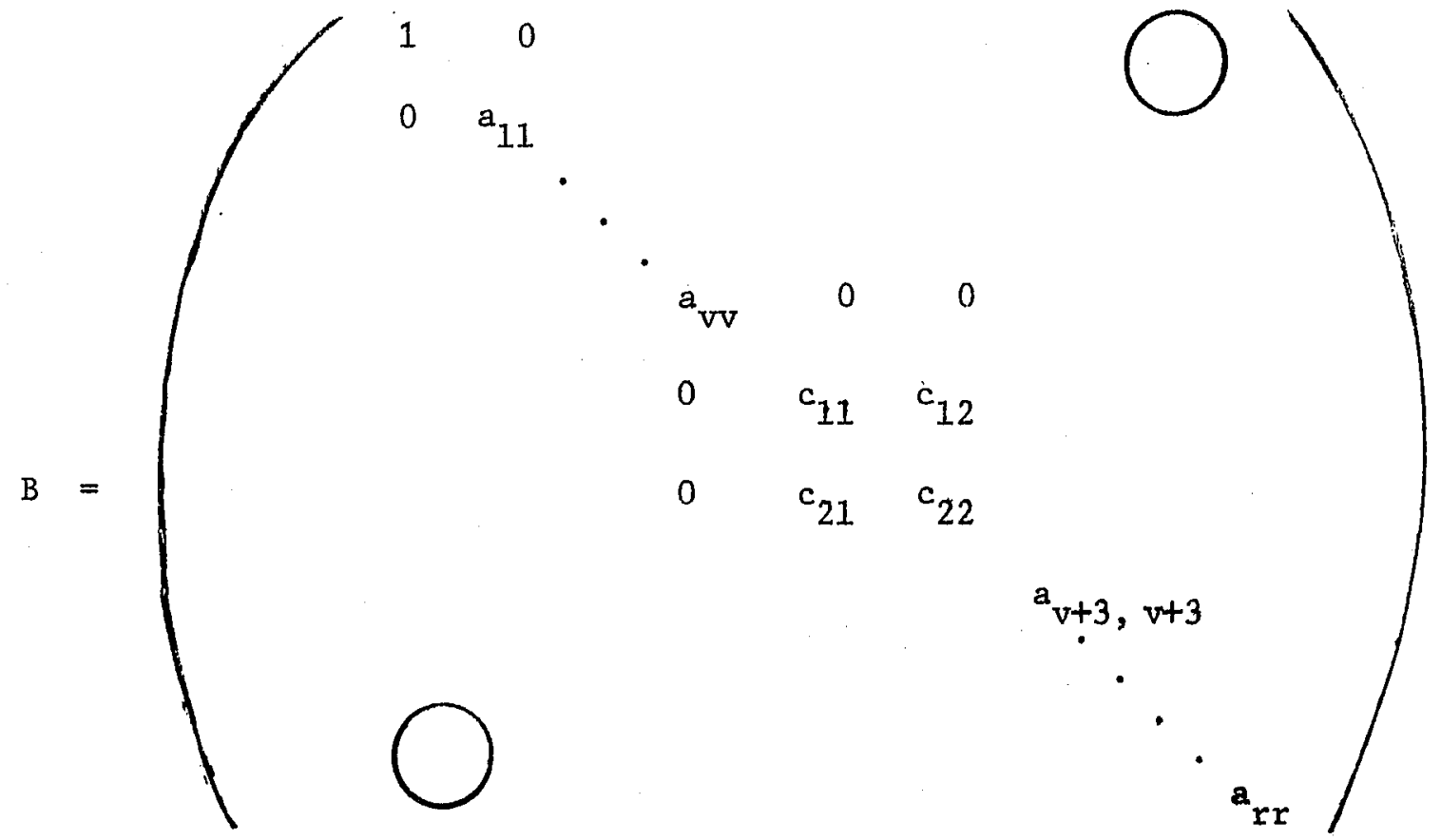

However, since only the $\left\{c_{i j}\right\}$ generate a continuum, the same simulation as before is involved. In carrying out these computations, the reader is reminded that if $\lambda_{2}$ is a repeated negative root, the simulation must be performed for all branches of $\log \lambda_{2}=\log \left|\lambda_{2}\right|+i(\pi \pm 2 \pi k)$ which satisfy Runnenberg's necessary condition for embeddability (equation 4.3). If $\lambda_{2}$ is a repeated 
positive root, the calculations must be carried out for all branches, except $k=0$, of $\log \lambda_{2}=\log \left|\lambda_{2}\right| \pm 2 \pi k 1$ which satisfy Runnenberg's condition. (The case $k=0$ can produce at most one version of $\log \hat{P} \varepsilon$ Q--see example 13). Example 14:

Recall the matrix of example 10 ,

$$
\hat{\mathrm{P}}=\frac{1}{3}\left(\begin{array}{lll}
1+2 \mathrm{x} & 1-\mathrm{x} & 1-\mathrm{x} \\
1-\mathrm{x} & 1+2 \mathrm{x} & 1-\mathrm{x} \\
1-\mathrm{x} & 1-\mathrm{x} & 1+2 \mathrm{x}
\end{array}\right)
$$

with $x=-e^{-2 \sqrt{3} \pi}$. This array is reduced to Jordan form by the similarity transformation

$$
H=\left(\begin{array}{ccc}
1 & 1 & 1 \\
1 & \frac{1}{2}(-1+\sqrt{3} i) & \frac{1}{2}(-1-\sqrt{3} i) \\
1 & \frac{1}{2}(-1-\sqrt{3} i) & \frac{1}{2}(-1+\sqrt{3} i)
\end{array}\right)
$$

Our problem is to indicate how a random sampling scheme of the type mentioned above could give some insight into the variety of branches of $\log \hat{P}$ which are in $\mathrm{Q}$. To illustrate the ideas, we restrict our consideration to the subset of matrices $B$ of the form

$$
B=\left(\begin{array}{ccc}
1 & 0 & 0 \\
0 & \beta & \alpha \\
0 & \alpha & \beta
\end{array}\right)
$$

where $\alpha$ and $\beta$ are arbitrary real numbers.

A computing strategy designed to identify matrices $B$ yielding branches of $\log \hat{\dot{P}} \varepsilon Q$ would begin by generating uniformly distributed $(\alpha, \beta)$ values within a square centered at the origin--the boundaries $|\alpha|=|\beta|=10$ are 
chosen here for illustrative purposes. Each generated value represents a point on the $\alpha-\beta$ plane for which $\log \hat{\mathrm{P}}=\mathrm{HB} \log \mathrm{J} \mathrm{B}^{-1} \mathrm{H}^{-1}$ is to be computed. If the resulting matrix is in $\underline{Q}$, a " + " is recorded at the point; otherwise a dot is recorded. In the present example, the flared pattern shown in figure 4, known as the "Iron Cross of the Red Baron (2nd class)," would result.

\section{F1gure 11 about here}

The restrictions on $\alpha$ and $\beta$ can be summarixed by the inequaltty $\frac{1}{2} \leq\left|\frac{\beta-\alpha}{\beta+\alpha}\right| \leq 2$. What is more to the point, the structure of the continuum is identical to the one reported in equation (3.23). (This may be verified by replacing $\left\{c_{i j}\right\}$ in [3.21] with the appropriate $\alpha$ and $\beta$ values, and computing the restrictions [3.22].) In general, however, by limiting $\left\{c_{i j}\right\}$ to real values only a portion of the continuum will be produced. 


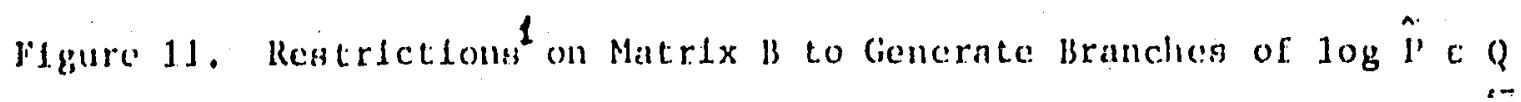

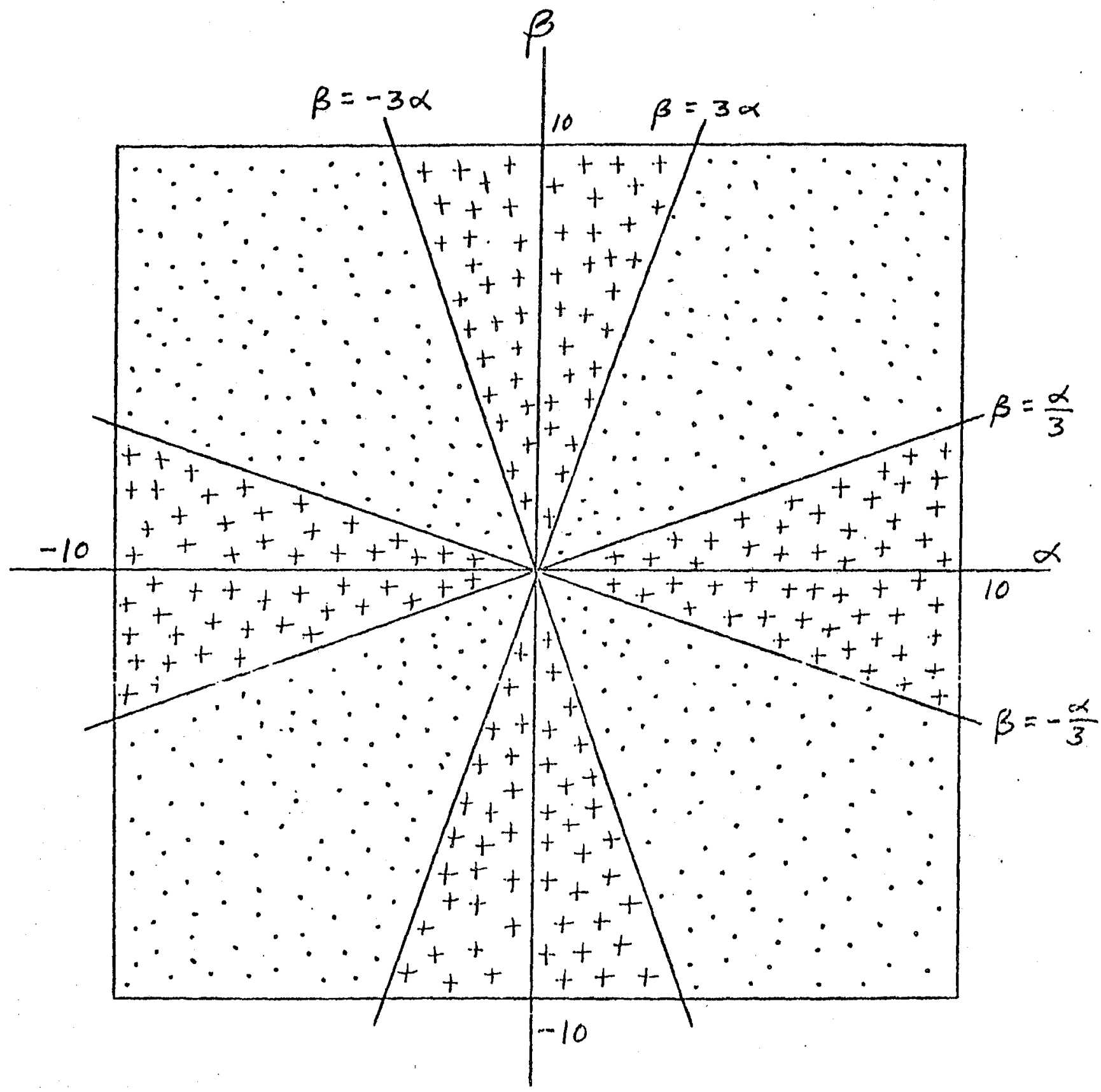

$1_{\text {The }}(\alpha,(i)$ valuess for which $\log \hat{p}$ : $Q$ are indicated by the symbol. "t.". The contours of constant values of log, $\hat{p}$ are the stmaght lines $\theta=\left(\frac{1+x}{1-x}\right) \alpha$, where: $\frac{1}{2} \leq|x| \leq 2$. 
FOOTNOTES

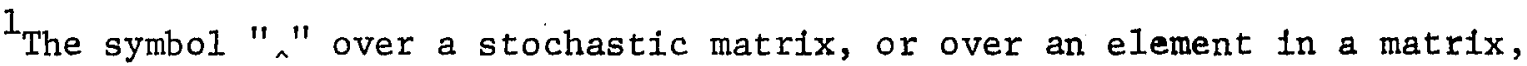
will mean that the quantity should be thought of as estimated directly from data. Matrices without this symbol should be viewed as obtained from a mathematical model.

${ }^{2}$ In most applications of Markov models, tests of this sort are not made. Hodge (1966) provides an exception.

${ }^{3}$ Where it is understood that the initial observation is at $t=0$ we will simplify our notation and write $\hat{P}\left(t_{1}\right)$, or even $\hat{P}$, In place of $\hat{P}\left(0, t_{1}\right)$.

${ }^{4}$ We wish to emphasize at the outset that our extensive criticlsm of estimation procedures used in Coleman's work in no way detracts from the utility of the mathematical formulations he employs, or from his strategies In translating sociological theory into mathematical statements. Indeed, his work has been a source of inspiration to both of us.

$5_{n_{1 j}}=$ number of persons starting in state 1 at a reference time $t=0$ who are in state $j$ at a later time $t=1 ; \mathfrak{n}_{1}=\sum_{j=1} \mathfrak{n}_{1 j}$. In our notation $\mathrm{n}_{j i} / \mathrm{n}_{j}=\hat{p}_{j 1}$. Actually, Coleman wrote $n_{i j} / n_{j}$. in place of $n_{j 1} / n_{j}$. This 1s obviously in error and elsewhere (Coleman 1964b, p. 4) he makes clear h1s intention.

6From the context, we interpret the word "asymptoticaliy" to mean monotone, rather than osc1llatory convergence.

${ }^{7}$ In different contexts we speak of checking whether $Q=\frac{1}{t} \log \hat{P} \varepsilon \underline{Q}$, or whether $\log \hat{P} \varepsilon \mathrm{Q}$. Because multiplication of a matrix by a real-valued quantity does not alter its character with respect to satisfying conditions (2.2), the two tests are equivalent. 
${ }^{8}$ These inequalities were established by F. I. Karpelewitsch (1951); they represent a considerable strengthening of the well known restriction that all eigenvalues of a stochastic matrix must lie inside the unit circle.

${ }^{9}$ For a lucid and detailed mathematical exposition, the reader should consult Gantmacher (1960, chap. 5).

${ }^{10} \mathrm{~A}$ function is said to be analytic at $\mathrm{x}$ if it has a derivative in a neighborhood containing the point.

${ }^{11}$ Although the Taylor series expansion has an infinite number of terms, $\left(J_{1}-\lambda_{1} I\right)^{n}=0$ for all values of $n \geq v_{i}$.

12 This matter is discussed in proposition 2.

${ }^{13}$ The simplest way to appreciate the multiple valued character of the logarithm is to begin with the definition: $x=10 g y$ if $x$ is a solution of the equation $e^{X}=y$ for a given $y$. Suppose $x$ is such a solution. Then, for any integer $k, e^{x+2 \pi k i}=e^{x} e^{2 \pi k i}=e^{x}=y\left(\right.$ since $\left.e^{2 \pi k i}=\cos 2 \pi k+1 \sin 2 \pi k=1\right)$. Therefore, log $\mathrm{y}$ takes on the values $\mathrm{x}, \mathrm{x} \pm 2 \pi, \mathrm{x} \pm 4 \pi$, etc.

${ }^{14}$ Al1 logarithms are to base e. The subscript $k$ denotes the branch number of the logarithm of a scalar quantity, and takes on the values $k=0, \pm 1$, $\pm 2, \ldots$. The subscript $\mathrm{K}$ denotes a version of the logarithm of a matrix, and specifies a combination of branches of the logarithm of the elgenvalues.

${ }^{15}$ Sylvester's formula has been effectively employed by S. Johansen (1974) In a recent study of the embedding problem. His results, however, are less general than the ones presented here because Sylvester's formula also provides less than a complete description of the logarithm of a matrix. This point is elaborated in proposition 2. 
${ }^{16}$ On computing the elementary divisors of a matrix consult Gantmacher (1960, pp. 139-145).

${ }^{17}$ When $r_{k}=1$ for $k=1, \ldots, m$, then (3.15) reduces to sylvester's formula (3.14).

18 The matrix (3.18) was introduced by J. Cuthbert (19̈73) In order to exhibit an example of a stochastic matrix compatible with a continuum of Markov models. Cuthbert's continuum arises when you choose $c_{11}=c_{22}=1$ and $c_{12}=c_{21}$, real. Then the constraints on $u$ and $v$ entail that $\left|c_{12}\right|=$ $\left|c_{21}\right| \leq 1 / 3$. This cholce does not, however, lead to all of the branches of $\log \hat{\mathrm{P}}$ given in (3.23), which represents an exhaustive list in $\mathrm{g}$.

If $\lambda=a+b i$, then $\rho=|\lambda|=\sqrt{a^{2}+b^{2}}$ and $\theta=\tan ^{-1} \frac{b}{a}$.

20 These formulas were computed from (3.24) by solving for $k$ (In the arctangent) at each bound.

$21_{k}$ may be positive or negative. The sign is chosen according to whether one wants to "move backward" to a repeated eigenvalue situation $(+k)$, or "move forward" $(-k)$. Note also that $k=0$ will not generate a continuum at $\theta=0$. These issues are addressed in greater detall in sections 4.2 and 4.3.

${ }^{22}$ For brevity in the discussion we focus on positive branches $(k>0)$. An analogous description can be presented for negative branches of the logarithm.

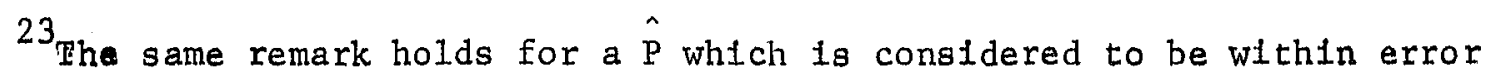
distance of $\hat{\mathrm{P}}\left(\frac{\mathrm{n} \pi}{\mathrm{b}}\right)$. 
${ }^{24}$ In connection with this point we refer the reader to the $4 \times 4$ matrix $\hat{P}\left(t_{1}\right)$ in example 12 . The reason why it is reasonably similar to the equilibrium matrix for the process can now be appreciated; namely, the complex conjugate eigenvalues are close to zero in magnitude.

25 This recommendation assumes that we have observed the flrst appearance of a continuum, which will be the most common situation. If we have observed the second occurrence, the times $t_{2}=\frac{k t}{2} 1$ should be avolded. If it is a third occurrence, omit the times $t_{2}=\frac{k t}{3} 1$, etc. As a practical guide, 1 a researcher avoids the two sets of time points cited in this footnote he is unlikely to encounter a second continuum.

${ }^{26}$ The Q-matrices in example 12 are the ones in this 11lustration multiplied by $t=1.05$.

${ }^{27}$ Because $L(t)=\{$ the number of branches of $\log P(t) \varepsilon \varrho\}$ is a nondecreasing function of time (except for isolated occurrences of continua), it is not necessary to examine points earlier than $t_{1}$.

${ }^{28}$ We recommend beginning this search in the 2-dimensional subspace defined by the conditions $c_{11}=c_{22}, c_{12}=c_{21}$, real. Then extend the search to the 4-dimensional space defined by the restriction that $\left\{c_{i j}\right\}$ be real, and finally introduce complex numbers in the full 8-dimensional space. Improved strategies for exploring this kind of continuum are currently in the preliminary development stage. 
Anderson, T. W. and Goodman, L. A.

1957 "Statistical inference about Markov chains." Annals of Mathematical Statistics $28: 89-109$.

Bartholomew, D. J.

1973 Stochastic Models for Soclal Processes (2nd edition). New York: Wiley.

Beshers, J. M. and E. O. Laumann

1967 "Social distance: A network approach." American Soclological

Review 32:225-236.

Billingsley, $\mathrm{P}$.

1961 Statistica1 Inference for Markov Processes. Chicago: University of Chicago Press.

Blumen, I., M. Kogan, and P. J. McCarthy.

1955 The Industrial Mobility of Labor as a Probability Process. Cornell Studies of Industrial and Labor Relations, Vol. 6. Ithaca, New York: Cornell University.

Boudon, R.

1973 Mathematical Structures of Soclal Mobl11ty. New York: American E1sevier Co.

Chung, K. L.

1967 Markov Chains with Stationary Transition Probabilities. Berlin: Springer.

Cipolla, M.

1932 "Sulle matrice espressione analitiche di un'altra." Rend, Circ. Mat. di Palermo 56:144-154. 
Cohen, B.

1963 Conflict and Conformity: A Probability Model and Its Application. Cambridge, Mass.: MIT Press.

Coleman, James S.

$1964 \mathrm{a}$ Introduction to Mathematical Sociology. New York: Free Press.

$1964 \mathrm{~b}$ Models of Change and Response Uncertainty. Englewood Cliffs, New Jersey: Prentice-Ha11.

1968 "The mathematical study of change." In Hubert M. and Ann B. Blalock (eds.), Methodology in Social Research. New York: McGrawHi11.

1973 The Mathematics of Collective Action. Chicago: Aldine. Cuthbert, J. R.

1972 "On uniqueness of the logarithm for Markov semi-groups." Journal of the London Mathematical Society 4:623-630.

1973 "The logarithm function for finite-state Markov semi-groups," Journal of the London Mathematical Society $6: 524-532$.

Elfving, $G$.

1937 "Zur Theorfe der Markoffschen ketten." Acta Social Science Fennicae n. Ser. A. 2, No. 8 .

Gantmacher, F. R.

1960 The Theory of Matrices (vol. 1). New York: Chelsea. Ginsberg, R.

1971 "Semi-Markov processes and mobility." Journal of Mathematical Sociology $1: 233-263$.

Hodge, R. W.

1966 "Occupational mobility as a probability process." Demography 3:19-34. 
Johansen, S.

1973 "A central limit theorem for finite semi-groups and 1ts application to the Imbedding problem for finite-state Markov chains." Zeltschrifft für Wahrscheinlichkeitstheorle $26: 171+190$.

1974 "Some results on the imbedding problem for finite Markov chains. Journal of London Mathematical Society 8:345-351.

Karpelewitsch, F. L.

1971 "On the characteristic roots of a matrix with non-negative elements." Isvestija. ser. mat. 15:361-383.

Kingman, J. F. C.

1962 "The imbedding problem for finite Markov chains." Zeitschrlfft für Wahrschein11chke1tstheorie $1: 14-24$.

Lleberson, S. and G. V. Fugultt

1967 "Negro-whtte occupational differences in the absence of discrimination." American Journal of Soctology $73: 188-200$.

Mayer, Thomas F.

1972 "Models of intra-generational mobility." In Joseph Berger, Morris Zeldich, Jr., and Bo Anderson, Soclological Theories in Progress. New York: Houghton-Miffin.

McDi11, Edward L., and James S. Coleman.

1963 "High school social status, college plans, and interest in academic achievement: A panel analysis. American Soclological Review 28 (December) : 905-18.

McFarland, David D.

1970 "Intra-generational social mobility as a Markov process: Including a time-stationary Markovian model that explains observed declines in mobility rates over time." American Sociological Review 35:463-476. 
Prais, S. J.

1955 "Measuring social mobility." Journal of the Royal Statistical Soclety 118 (Series A):55-66.

Rinehart, R. F.

1955 "The equivalence of definitions of a matric function." American Mathematical Monthly $62: 395-414$.

Runnenberg, J. Th.

1962 "On Elfving's problem of imbedding a time-discrete Markov chain in a continuous time one for finitely many states." Indag. Math. 24: 536-541.

Singer, Burton and Seymour Spilerman.

1974 "Social mobility models for heterogeneous populations." In Herbert L. Costner (ed.), Soctological Methodology 1973-1974. San Francisco: Jossey-Boss.

Spilerman, Seymour.

1972a "The analysis of mobility processes by the introduction of Independent variables into a Markov chain." American Sociological Review 37 (June) : 277-94.

1972b "Extensions of the mover-stayer mode1." Amer1can Journal of Soclology $78: 599-627$.

Sylvester, J. J.

1883 "On the equation to the secular inequalities in the planetary theory." Philosophical Magazine 16:267-269.

Wolfgang, Marvin E., Robert M. Figlio, and Thorsten Sellin. 1972 Delinquency in a Birth Cohort. Chicago: University of Chicago Press. Zah1, Samuel.

1955 "A Markov process model for follow-up studies." Human Biology 27: $90-120$. 